ESAIM: COCV 21 (2015) 165-189

DOI: $10.1051 / \mathrm{cocv} / 2014021$
ESAIM: Control, Optimisation and Calculus of Variations

www.esaim-cocv.org

\title{
UNCONSTRAINED VARIATIONAL PRINCIPLES FOR LINEAR ELLIPTIC EIGENPROBLEMS *
}

\author{
G. Auchmuty ${ }^{1}$ And M.A. Rivas ${ }^{1}$
}

\begin{abstract}
This paper introduces and studies some unconstrained variational principles for finding eigenvalues, and associated eigenvectors, of a pair of bilinear forms $(a, m)$ on a Hilbert space $V$. The functionals involve a parameter $\mu$ and are smooth with well-defined second variations. Their non-zero critical points are eigenvectors of $(a, m)$ with associated eigenvalues given by specific formulae. There is an associated Morse-index theory that characterizes the eigenvector as being associated with the $j$ th eigenvalue. The requirements imposed on the forms $(a, m)$ are appropriate for studying elliptic eigenproblems in Hilbert-Sobolev spaces, including problems with indefinite weights. The general results are illustrated by analyses of specific eigenproblems for second order elliptic Robin, Steklov and general eigenproblems.
\end{abstract}

Mathematics Subject Classification. 35P15, 49R05, 58E05.

Received October 3, 2013.

Published online December 3, 2014.

\section{INTRODUCTION}

This paper describes a family of unconstrained variational principles for finding eigenvalues and eigenvectors of a pair $(a, m)$ of continuous symmetric bilinear forms on a real separable Hilbert space $V$. The requirements on the forms $a, m$ are appropriate for elliptic eigenproblems set in Hilbert-Sobolev spaces on bounded regions. The framework is quite general but, in Sections $8-11$, the variational principles for some Robin, Steklov and general eigenproblems for second order elliptic equations on bounded regions in $\mathbb{R}^{N}$ are detailed.

The functionals have well-defined first and second variations and their non-zero critical points are eigenvectors of $(a, m)$. When $\mu$ is large enough, their global minimizers occur at critical points associated with the least eigenvalue of $(a, m)$. For fixed $\mu$, the critical points of the functional $\mathscr{G}(\cdot, \mu)$ are associated with the smallest $J$ eigenvalues of $(a, m)$ with $J$ finite. Since the functionals are smooth, the second variation at a non-degenerate critical point has a well-defined Morse index. The Morse index counts the number of eigenvalues that are less than the current eigenvalue.

This analysis may well be compared with the corresponding analysis for constrained variational principles using Rayleigh's principle. Such theories are described in Chapter 8 of Attouch et al. [1] and also in the

Keywords and phrases. Robin eigenproblems, Steklov eigenproblems, Morse indices, unconstrained variational problems.

* This research was partially supported by NSF award DMS 1108754.

1 Department of Mathematics, University of Houston, Houston, Tx 77204-3008, USA.

auchmuty@uh.edu; mauricio@math.uh.edu 
monograph of Edmunds and Evans [10]. An analysis of elliptic eigenproblems based on the use of two real Hilbert spaces $V, H$ may be found in Blanchard and Brüning [8], Chapter 6. In contrast to most of these references the analysis here only involves continuous bilinear forms on the real Hilbert space $\mathrm{V}$ and avoids the introduction of dual, or other, Sobolev spaces and linear transformations. This framework allows the application to more general boundary conditions and eigenproblems than these texts.

The forms generally include boundary integrals with the eigenvalue equation being the weak form of the usual elliptic problem. The basic assumptions are that $a$ is continuous, symmetric and $V$-coercive on the real Hilbert space $V$ and $m$ is symmetric, weakly continuous and positive on $V$. A description of the Rayleigh principle analysis based on continuous bilinear forms may be found in Auchmuty [6] and results from that paper are reprised in Section 2. Section 3 describes some basic results about (Morse) indices and the decomposition of bilinear forms.

The unconstrained variational principle is introduced in Section 4 where some basic properties of the functional are proved. This enables results about the existence of global minimizers of the variational problem and the characterization of critical points as eigenvectors of the pair $(a, m)$. Moreover, a bifurcation diagram for the dependence of critical points on $\mu$ is provided.

Section 5 describes the evaluation of the Morse index and null index of critical points of the functional and shows that non-degenerate critical points are precisely those associated with simple eigenvalues of $(a, m)$. In particular, non-degenerate critical points of $\mathscr{G}(\cdot, \mu)$ corresponding to the $j$ th eigenvalue (counting multiplicity) are saddle points of $\mathscr{G}(\cdot, \mu)$ of Morse index $(j-1)$ provided $\mu$ is large enough.

In Section 6, it is shown how an appropriate penalization of the functional $\mathscr{G}(\cdot, \mu)$ provides a functional whose unconstrained minimizer occurs at a critical point associated with the second eigenvalue of $(a, m)$.

In Section 7 some of the preceding results are extended to the case where the bilinear form $m$ is allowed to have varying sign. In this indefinite case, the variational principle for the positive eigenvalues and that for the negative eigenvalues are different.

The last sections of this paper illustrate the application of this general theory to some different eigenproblems for second-order divergence form elliptic problems. Section 8 treats problems with homogeneous (zero) Robin boundary conditions. 9 treats Steklov eigenproblems that involve homogeneous equations with the eigenparameter only in the boundary condition. 10 describes results for these variational principles for Steklov eigenproblems with indefinite weight functions. Finally, Section 11 describes results for eigenproblems where the eigenparameter enters both the differential equation and the boundary condition.

Some of the results here are related to results obtained by the first author on unconstrained variational principles for various other eigenproblems described in $[2,6]$.

\section{BILINEAR FORMS AND NOTATION}

Throughout this paper $V$ is a real, separable, infinite dimensional, Hilbert space. The inner product and norm on $V$ are denoted $\langle\cdot, \cdot\rangle_{V}$ and $\|\cdot\|_{V}$. The dual space of $V$ will be denoted $V^{*}$ and is again a separable infinite dimensional Hilbert space with the usual dual norm and inner product. Through Section 7 our treatment is quite general but the aim is to provide the results appropriate for the examples studied thereafter that involve eigenproblems for elliptic forms on $H^{1}(\Omega)$.

A bilinear form $b: V \times V \rightarrow \mathbb{R}$ is said to be symmetric if $b(u, v)=b(v, u)$ for all $(u, v) \in V \times V$. Our interest is in finding non-trivial solutions $(\lambda, u) \in \mathbb{R} \times V$ of

$$
a(u, v)=\lambda m(u, v) \text { for all } v \in V .
$$

Here $a: V \times V \rightarrow \mathbb{R}$ and $m: V \times V \rightarrow \mathbb{R}$ are continuous symmetric bilinear forms on $\mathrm{V}$. This will be called the $(a, m)$ eigenproblem. The number $\lambda$ is an eigenvalue of $(a, m)$ if there is a non-zero vector $u \in V$ satisfying $(2.1)$. Any such $u$ is called an eigenvector of $(a, m)$ corresponding to $\lambda$.

When $\lambda$ is an eigenvalue, let $E_{\lambda}$ be the set of all $u \in V$ such that (2.1) holds. An eigenvector $e$ of $(a, m)$ is said to be $m$-normalized provided $m(e, e)=1$. The number of linearly independent eigenvectors of $(a, m)$ 
corresponding to the eigenvalue $\lambda$ is called the multiplicity of $\lambda$. When the multiplicity of $\lambda$ is one, then $\lambda$ is said to be a simple eigenvalue.

Define $\mathscr{A}, \mathscr{M}$ to be the quadratic forms on $\mathrm{V}$ associated with $(a, m)$, respectively, so that

$$
\mathscr{A}(u):=a(u, u) \text {, and } \mathscr{M}(u):=m(u, u) .
$$

Our results about the eigenproblem for $(a, m)$ will be proved using variational methods subject to some of the following conditions.

(A1) $a(\cdot, \cdot)$ is a continuous symmetric bilinear form that also is $V$-coercive. That is there are $0<k_{0} \leq k_{1}<\infty$ such that

$$
k_{0}\|u\|_{V}^{2} \leq \mathscr{A}(u) \leq k_{1}\|u\|_{V}^{2} \quad \text { for all } \quad u \in V .
$$

(A2) $m(\cdot, \cdot)$ is a weakly continuous symmetric bilinear form on $V$.

(A3) $\mathscr{M}(u) \geq 0$ for all $u$ in $V$ and $\mathscr{M}(u)>0$ for some $u$ in $V$.

(A4) $\mathscr{M}(u)>0$ for all non-zero $u$ in $V$.

When (A1) holds then the bilinear form $a(\cdot, \cdot)$ defines an inner product on $V$ that is equivalent to the $V$ inner product. The functional $\mathscr{M}$ is said to be positive when (A3) holds and then $\|u\|_{m}:=\sqrt{\mathscr{M}(u)}$ will be a semi-norm on $V$. When $\mathscr{M}$ satisfies (A4) it is said to be strictly positive and $m(\cdot, \cdot)$ is an inner product on $V$.

If (A1) - (A3) hold let $v=u$ in (2.1) to conclude that every eigenvalue $\lambda$ must be strictly positive and the eigenvectors obey $m(u, u)>0$.

When $b$ is a symmetric continuous bilinear form on $V$, then the null space $N(b)$ of $b$ is the set of all vectors $v \in V$ satisfying

$$
b(v, w)=0 \quad \text { for all } \quad w \in V .
$$

$b$ is said to be non-degenerate if $N(b)=\{0\}$; otherwise, $b$ is said to be degenerate. The dimension of $N(b)$ is called the null index of $b$ and denoted $i_{0}(b)$. Moreover, we say $b$ has finite rank $M$, with $M \in \mathbb{N}$, provided there are $M$ linearly independent functionals $f_{1}, \ldots, f_{M}$ in $V^{*}$ with

$$
b(u, v)=\sum_{j=1}^{M} f_{j}(u) f_{j}(v) \quad \text { for all } \quad u, v \in V .
$$

Two vectors $u, v \in V$ are said to be $b$-orthogonal provided $b(u, v)=0$. A subset $\mathscr{E}$ of $V$ is $b$-orthonormal provided any two vectors in $\mathscr{E}$ are $b$-orthogonal and $b(e, e)=1$ for each $e \in \mathscr{E}$. A subset $\mathcal{E}$ of $V$ is said to be a basis of $V$ if it is a maximal linearly independent set in $V$ with respect to inclusion. $\mathcal{E}$ is a b-orthogonal basis of $V$ when $\mathcal{E}$ is a basis that also is b-orthogonal. Note that if $e$ is an eigenvector of (2.1) corresponding to an eigenvalue $\lambda \neq 0$, then

$$
m(u, e)=0 \Longleftrightarrow a(u, e)=0 .
$$

In particular if $e_{j}, e_{k}$ are eigenvectors of $(a, m)$ corresponding to distinct eigenvalues $\lambda_{j}, \lambda_{k}$, then they are both $a$-orthogonal and $m$-orthogonal.

When (A1)-(A3) hold, let $N(m)$ be the null space of $m$ and $W$ be its orthogonal complement so

$$
V=N(m) \oplus_{a} W
$$

is an orthogonal decomposition of $V$ with respect to the $a$-inner product. The eigenvectors of $(a, m)$ lie in $W$.

When (A1)-(A3) hold, there are eigenvalues and eigenvectors of $(a, m)$. Specifically the following result is a restatement of results described in Auchmuty [6]. 
Theorem 2.1. Assume the pair (a,m) satisfies (A1)-(A3). Then either

(i) there are finitely many m-orthonormal eigenvectors $\mathcal{E}_{N}:=\left\{e_{j}: 1 \leq j \leq N\right\}$ corresponding to non-zero eigenvalues $\lambda_{1} \leq \lambda_{2} \leq \ldots \leq \lambda_{N}$ of $(a, m)$, or;

(ii) there are countably infinitely many m-orthonormal eigenvectors $\mathcal{E}:=\left\{e_{j}: j \geq 1\right\}$ corresponding to non-zero eigenvalues $\lambda_{1} \leq \lambda_{2} \leq \ldots$ of the pair $(a, m)$, with no finite accumulation point.

In both cases, these eigenvalues can be found iteratively and are repeated according to multiplicity. When (A4) holds, then (ii) holds, $\lambda_{1}>0$ and $\mathcal{E}$ is an m-orthonormal basis of $V$.

Proof. The assumptions on the bilinear forms $a(\cdot, \cdot)$ and $m(\cdot, \cdot)$ are the requirements for the bilinear forms in Theorem 4.2 and Theorem 4.3 in Auchmuty [6]. Those theorems yield the desired results with $a$-orthonormality in place of $m$-orthonormality. As (2.5) holds for an eigenvector $e$ corresponding to non-zero eigenvalue $\lambda$ of $(a, m), a$-orthogonality may be replaced by $m$-orthogonality. Moreover, $m$-normalized eigenvectors are obtained from $a$-normalized eigenvectors by taking $\tilde{e}:=\lambda^{1 / 2} e$ for an $a$-normalized eigenvector $e$ corresponding to the eigenvalue $\lambda$.

In this paper, various results from the calculus of variations will be used. Background material may be found in Attouch, Buttazzo, and Michaille [1], Blanchard and Brüning [8] or Zeidler [12].

Let $\mathscr{F}: V \rightarrow \mathbb{R}$ be a given functional. The first variation of $\mathscr{F}$ at a point $u \in V$ in the direction $v \in V$ is defined by

$$
\delta \mathscr{F}(u ; v):=\left.\frac{\mathrm{d}}{\mathrm{d} t} \mathscr{F}(u+t v)\right|_{t=0}
$$

when this derivative exists. When $\delta \mathscr{F}(u ; v)$ exists for all $v \in V$ and is a continuous linear form in $v$, then $\mathscr{F}$ is said to be Gâteaux differentiable at $u$ and the linear functional $v \mapsto \delta \mathscr{F}(u ; v)$ is the Gâteaux derivative of $\mathscr{F}$ at $u$.

A point $u \in V$ is a critical point of $\mathscr{F}$ provided $\mathscr{F}$ is Gâteaux differentiable at $u$ and

$$
\delta \mathscr{F}(u ; v)=0 \text { for all } v \in V .
$$

A number $c$ is a critical value of $\mathscr{F}$ if there is a critical point $u$ with $\mathscr{F}(u)=c$. The second variation of $\mathscr{F}$ at $u$ in the directions $v, w \in V$ is defined by

$$
\delta^{2} \mathscr{F}(u ; v, w):=\left.\frac{\partial^{2}}{\partial t_{2} \partial t_{1}} \mathscr{F}\left(u+t_{1} v+t_{2} w\right)\right|_{t_{1}=t_{2}=0}
$$

whenever this derivative exists. If $\delta^{2} \mathscr{F}(u ; v, w)$ exists for all $v, w \in V$ and is a continuous bilinear form in $(v, w)$, then $\mathscr{F}$ is said to be twice Gâteaux differentiable at $u$ and the bilinear form $(v, w) \mapsto \delta^{2} \mathscr{F}(u ; v, w)$ is called the Hessian form of the functional $\mathscr{F}$ at $u$.

\section{EIGENPROBLEMS AND INDICES}

In this paper we shall prove results about the critical points of smooth unconstrained variational problems, the critical points yielding solutions of certain eigenvalue problems. In particular the Morse index of a critical point will be related to an ordering of the eigenvalues of $(a, m)$. First the Morse index of forms that satisfy a Gårding type inequality will be defined. This proceeds along the lines outlined in Zeidler ([12], Sect. 37.27b) but uses bilinear forms directly - not associated linear operators between dual spaces.

Assume that $b$ satisfies:

(M1) $b: V \times V \rightarrow \mathbb{R}$ is a continuous symmetric bilinear form, and 
(M2) there is a bilinear form $m$ satisfying (A2), (A4) and $k_{2}, k_{3}>0$ such that

$$
\mathscr{B}(v):=b(v, v) \geq k_{2}\|v\|_{V}^{2}-k_{3} m(v, v) \text { for all } v \in V .
$$

The Morse index $i(b)$ of a form $b$ is defined to be the maximal dimension of subspaces $W$ of $V$ on which $\mathscr{B}$ is strictly negative.

The following results show that the Morse index and the null index of $b$ are finite and independent of $m$. In particular these indices are related to the eigenproblem for $(b, m)$ :

$$
b(v, w)=\lambda m(v, w) \text { for all } w \in V .
$$

In this section eigenvalues are counted with multiplicities. Note that the proof of Theorem 2.1 was based on a constructive algorithm in $[6]$ that determined successive eigenvalues.

Theorem 3.1. Assume the pair (b, $m$ ) satisfies (M1), (M2). Then the Morse index of $b$ is the number of negative eigenvalues of (3.2) counting multiplicities and is finite. The null index of $b$ is the multiplicity of 0 as an eigenvalue of (3.2) and is finite.

Proof. From (M2) we have $\mathscr{B}(v)+k_{3} \mathscr{M}(v) \geq k_{2}\|v\|_{V}^{2}$ for all $v \in V$, i.e., the bilinear form $\tilde{b}:=b+k_{3} m$ is $V$-coercive and thus satisfies the properties of the bilinear form in (A1). Since $m$ satisfies (A2) and (A4), by Theorem 2.1 there is an $m$-orthonormal basis $\mathcal{E}:=\left\{e_{j}: j \geq 1\right\}$ of $V$ consisting of eigenvectors for the pair $(\tilde{b}, m)$ corresponding to strictly positive eigenvalues $\tilde{\lambda}_{1} \leq \tilde{\lambda}_{2} \leq \ldots$ with $\lim _{j \rightarrow \infty} \tilde{\lambda}_{j}=\infty$. That is,

$$
\tilde{b}\left(e_{j}, v\right)=\tilde{\lambda}_{j} m\left(e_{j}, v\right) \text { for all } v \in V,
$$

and $m\left(e_{j}, e_{k}\right)=\delta_{j k}$ for all $j, k \in \mathbb{N}$. Taking $\lambda_{j}:=\tilde{\lambda}_{j}-k_{3}$ yields

$$
b\left(e_{j}, v\right)=\lambda_{j} m\left(e_{j}, v\right) \quad \text { for all } \quad v \in V,
$$

so that $\mathcal{E}$ is an $m$-orthonormal basis for $V$ consisting of eigenvectors of the pair $(b, m)$ corresponding to the eigenvalues $-\infty<\lambda_{1} \leq \lambda_{2} \leq \ldots$ with $\lim _{j \rightarrow \infty} \lambda_{j}=\infty$. Hence, for each $v \in V$ we have

$$
v=\sum_{j=1}^{\infty} m\left(v, e_{j}\right) e_{j} \quad \text { and } \quad b(v, w)=\sum_{j=1}^{\infty} \lambda_{j} m\left(v, e_{j}\right) m\left(w, e_{j}\right) .
$$

Let

$$
W_{-}=\operatorname{span}\left\{e_{j}: \lambda_{j}<0\right\} \text { and } W_{0}=\operatorname{span}\left\{e_{j}: \lambda_{j}=0\right\} .
$$

The dimensions of $W_{-}$and $W_{0}$ are both finite as the $\lambda_{j}$ 's form an increasing sequence of real numbers with no finite accumulation point. The representation of $b$ in (3.3) yields

$$
\mathscr{B}(v)=\sum_{j=1}^{\infty} \lambda_{j} m\left(e_{j}, v\right)^{2},
$$

so $W_{-}$is a subspace of maximal dimension on which $\mathscr{B}$ is strictly negative and the first assertion holds. From (3.3) we also have $v \in W_{0} \Longleftrightarrow v \in N(b)$ so the second assertion holds.

The next result shows that the Morse index of $b$ is independent of the choice of $m$ satisfying (A2), (A4). This may be regarded as an infinite-dimensional version of Sylvester's law of inertia for finite-dimensional quadratic forms. 
Theorem 3.2. Assume $b$ satisfies (M1), $m_{1}, m_{2}$ both satisfy (A2), (A4) and (b, $\left.m_{1}\right)$ and $\left(b, m_{2}\right)$ both satisfy inequalities of the form (3.1). Then:

(i) the number of negative eigenvalues of $\left(b, m_{1}\right)$ equals the number of negative eigenvalues of $\left(b, m_{2}\right)$, and,

(ii) the multiplicity of 0 as an eigenvalue of $\left(b, m_{1}\right)$ equals the multiplicity of 0 as an eigenvalue of $\left(b, m_{2}\right)$.

Proof. Denote by $i\left(b, m_{1}\right)$ the number of negative eigenvalues of $\left(b, m_{1}\right)$. By Theorem $3.1 i\left(b, m_{1}\right)$ is finite, and the dimension of any subspace $W$ on which $\mathscr{B}$ is strictly negative satisfies $\operatorname{dim} \mathrm{W} \leq i\left(b, m_{1}\right)$. Let $W_{-}$be the subspace of $V$ generated by eigenvectors corresponding to negative eigenvalues of $\left(b, m_{2}\right)$. By Theorem 3.1 again, $W_{-}$has finite dimension, denoted $i\left(b, m_{2}\right)$, equal to the number of negative eigenvalues of $\left(b, m_{2}\right)$. Since $\mathscr{B}$ is strictly negative on $W_{-}$this implies $i\left(b, m_{2}\right) \leq i\left(b, m_{1}\right)$.

Interchanging the roles of $i\left(b, m_{1}\right)$ and $i\left(b, m_{2}\right)$ gives the reverse inequality so (i) holds. Assertion (ii) holds as the equation for the null eigenvectors is independent of $m$.

A consequence of these theorems is the following decomposition result.

Corollary 3.3. Assume the pair (b, $m$ ) satisfies (M1), (M2) and $b$ is non-degenerate. Then there are continuous symmetric bilinear forms $b_{-}$and $b_{+}$such that $b=b_{+}-b_{-}$with $b_{-}$having finite rank equal to the Morse index of b. The corresponding quadratic forms satisfy $\mathscr{B}(v)=\mathscr{B}_{+}(v)-\mathscr{B}_{-}(v)$, with $\mathscr{B}_{+}$and $\mathscr{B}_{-}$being positive, convex functionals on $V$ and $\mathscr{B}_{-}$weakly continuous.

Proof. By Theorem 3.1 a subspace of maximal dimension on which $\mathscr{B}$ is strictly negative is spanned by $\mathcal{E}_{-}:=$ $\left\{e_{j}: 1 \leq j \leq i(b)\right\}$, where $i(b)$ is the (finite) Morse index of $b$ and the $e_{j}$ 's are $m$-orthonormal eigenvectors associated to strictly negative eigenvalues $\lambda_{j}$ of $(b, m)$.

Define the bilinear form $b_{-}: V \times V \rightarrow \mathbb{R}$ by

$$
b_{-}(v, w):=-\sum_{j=1}^{i(b)} \lambda_{j} m\left(e_{j}, v\right) m\left(e_{j}, w\right) .
$$

Then $b_{-}$has finite rank $i(b)$, so (3.3) and (3.4) imply that the bilinear form $b_{+}:=b-b_{-}$is continuous and symmetric on $V$, so the first assertion holds.

From (3.4), the quadratic form $\mathscr{B}_{-}(v):=b_{-}(v, v)$ is a positive, convex functional on $V$. It is weakly continuous as $b_{-}$has finite rank. From (3.3) and (3.4), the quadratic form $\mathscr{B}_{+}$corresponding to $b_{+}$also is a positive, convex functional on $V$ so the second assertion holds.

That is, each $m$ satisfying (M2) yields a different decomposition $b=b_{+}-b_{-}$, but the rank of $b_{-}$is independent of the choice of $m$. This rank is the Morse index $i(b)$ of $b$ on $V$.

To prove results about the critical points of the unconstrained variational problems, the following terminology is used.

Let $\mathscr{F}: V \rightarrow \mathbb{R}$ be a functional that is twice Gâteaux differentiable on $V$. A critical point $u$ of $\mathscr{F}$ is said to be degenerate or non-degenerate accordingly as the Hessian form $\delta^{2} \mathscr{F}(u ; \cdot, \cdot)$ is a degenerate or non-degenerate bilinear form. The Morse index and null index of a critical point $u$ of $\mathscr{F}$ are defined to be the Morse index and null index of the Hessian form $\delta^{2} \mathscr{F}(u ; \cdot, \cdot)$, and are denoted $i(u)$ and $i_{0}(u)$, respectively.

In the following sections, the Morse and null indices of the Hessian forms $h_{u}(\cdot, \cdot):=\delta^{2} \mathscr{F}(u ; \cdot, \cdot)$ of functionals at critical points will be evaluated. The preceding corollary is used to investigate the splitting of the quadratic form $\mathscr{H}_{u}(v):=h_{u}(v, v)$ into positive and negative parts. 


\section{UNCONSTRAINED VARIATIONAL PRINCIPLES FOR THE LEAST EIGENVALUE}

The standard variational principles for eigenvalues of symmetric matrices, bilinear forms and self-adjoint linear operators have been based on Rayleigh's principle which involves minimizing or maximizing the Rayleigh Quotient. This is essentially a constrained optimization problem. Auchmuty in $[3,6]$, has described some unconstrained variational principles for eigenproblems of matrices and compact linear operators. Here an unconstrained variational principle that applies to bilinear forms satisfying the Gårding type inequality (M2) will be investigated.

Define the functional $\mathscr{G}: V \times(0, \infty) \rightarrow \mathbb{R}$ by

$$
\mathscr{G}(u, \mu):=\mathscr{A}(u)-\mu \mathscr{M}(u)+\frac{1}{2} \mathscr{M}(u)^{2}
$$

where $\mathscr{A}, \mathscr{M}$ are given by (2.2).

Consider the unconstrained variational problem $\left(\mathcal{P}_{\mu}\right)$ of minimizing $\mathscr{G}(\cdot, \mu)$ on $V$ and finding

$$
\alpha(\mu):=\inf _{u \in V} \mathscr{G}(u, \mu) .
$$

This family of unconstrained variational principles has non-zero critical points that are eigenvectors of $(a, m)$ and a value that depends on the least eigenvalue of $(a, m)$. Some essential properties of this functional may be summarized as follows.

Theorem 4.1. Assume (A1)-(A2) hold, and $\mathscr{G}(\cdot, \mu)$ is defined by (4.1). Then

(i) $\mathscr{G}(\cdot, \mu)$ is continuous, coercive and weakly l.s.c. on $V$,

(ii) $\mathscr{G}(\cdot, \mu)$ is Gâteaux differentiable at each $u \in V$ with first variation at u given by

$$
\delta \mathscr{G}(u ; v ; \mu)=2 a(u, v)+2[m(u, u)-\mu] m(u, v),
$$

with direction vector $v$ in $V$, and

(iii) $\mathscr{G}(\cdot, \mu)$ has second variation at $u$ in the directions $v, w$ in $V$ given by

$$
\delta^{2} \mathscr{G}(u ; v, w ; \mu)=2 a(v, w)+2[m(u, u)-\mu] m(v, w)+4 m(u, v) m(u, w) .
$$

Proof. By assumption $\mathscr{A}, \mathscr{M}$ are continuous on $V$ so $\mathscr{G}(\cdot, \mu)$ is continuous on $V$. $\mathscr{A}$ is weakly l.s.c. as it is convex from (A1) so $\mathscr{G}(\cdot, \mu)$ is weakly l.s.c. as $\mathscr{M}$ is weakly continuous on $V$.

Now (2.3) and completing the square in (4.1) yields

$$
\mathscr{G}(u, \mu) \geq k_{0}\|u\|_{V}^{2}-\frac{\mu^{2}}{2} .
$$

Thus $\mathscr{G}$ is coercive on $V$.

Straightforward calculations show that the first variations of $\mathscr{A}, \mathscr{M}$ at $u$ are

$$
\delta \mathscr{A}(u ; v)=2 a(u, v), \quad \delta \mathscr{M}(u ; v)=2 m(u, v) .
$$

Then use of the chain rule on the last term in (4.1) leads to (4.3).

The second variations of $\mathscr{A}, \mathscr{M}$ are easily seen to be

$$
\delta^{2} \mathscr{A}(u ; v, w)=2 a(v, w) \quad \delta^{2} \mathscr{M}(u ; v, w)=2 m(v, w)
$$

as the functionals are quadratic and thus (4.4) follows. 
Property (ii) for the functional $\mathscr{G}(\cdot, \mu)$ shows that 0 is always a critical point of $\mathscr{G}(\cdot, \mu)$. A non-zero vector $u \in V$ is a critical point of $\mathscr{G}(\cdot, \mu)$ on $V$ if and only if $u$ is an eigenvector of $(a, m)$ corresponding to the eigenvalue $\lambda=\mu-m(u, u)$.

When (A1)-(A3) hold, the only possible eigenvalues of (2.1) are positive. Let $\lambda_{1}$ be the least eigenvalue of $(a, m)$ in this case, which exists and is finite from Theorem 2.1. Then the following result holds.

Theorem 4.2. Assume (A1)-(A3) hold, and $\mathscr{G}$ is defined by (4.1). If $\mu \leq \lambda_{1}$, then 0 is the unique critical point of $\mathscr{G}(\cdot, \mu)$ on $V$ and it is the unique minimizer of $\mathscr{G}(\cdot, \mu)$ with $\alpha(\mu)=0$.

Proof. From part (i) of Theorem 4.1, $\mathscr{G}(\cdot, \mu)$ attains a finite infimum on $V$, which by part (ii) must occur at a critical point $u$ which satisfies

$$
a(u, v)=[\mu-m(u, u)] m(u, v)
$$

for all $v \in V$. Since $\mathscr{M}$ is positive on $V$, this says that if $u \neq 0$ it must be an eigenvector of $(a, m)$ with eigenvalue $\lambda<\mu$. This is impossible if $\mu \leq \lambda_{1}$, so that $u=0$ is the only critical point of $\mathscr{G}(\cdot, \mu)$ on $V$. Thus the minimizer of $\mathscr{G}(\cdot, \mu)$ is $u=0$, and $\alpha(\mu)=\mathscr{G}(0, \mu)=0$.

When $\mu>\lambda_{1}$, however, there will be a non-zero minimizer of $\mathscr{G}(\cdot, \mu)$ and the following results about this minimization problem hold.

Theorem 4.3. Assume (A1)-(A3) hold, and $\mathscr{G}$ is defined by (4.1). If $\mu>\lambda_{1}$, then:

(i) the non-zero critical points of $\mathscr{G}(\cdot, \mu)$ are $\left(\mu-\lambda_{j}\right)^{1 / 2}$ e where $\lambda_{j}$ is an eigenvalue of $(a, m)$ with $\lambda_{j} \in(0, \mu)$ and $e$ an associated $m$-normalized eigenvector,

(ii) the minimizers of $\mathscr{G}(\cdot, \mu)$ on $V$ are $\left(\mu-\lambda_{1}\right)^{1 / 2} e_{1}$ where $e_{1}$ is an m-normalized eigenvector of $(a, m)$ associated with the least eigenvalue $\lambda_{1}$, and $2 \alpha(\mu)=-\left(\mu-\lambda_{1}\right)^{2}$.

Proof. From (4.5), non-zero critical points of $\mathscr{G}(\cdot, \mu)$ are eigenvectors $\tilde{u}$ corresponding to eigenvalues $\lambda_{j}=$ $\mu-m(\tilde{u}, \tilde{u})$ of $(a, m)$. Substituting this $\tilde{u}$ into (4.5) yields

$$
a(\tilde{u}, \tilde{u})=[\mu-m(\tilde{u}, \tilde{u})] m(\tilde{u}, \tilde{u})=\lambda_{j}\left(\mu-\lambda_{j}\right) .
$$

The positivity of $\mathscr{A}$ implies that $\lambda_{j}\left(\mu-\lambda_{j}\right)>0$ as $\tilde{u} \neq 0$. Thus $\lambda_{j}<\mu$, and $\lambda_{j} \in(0, \mu)$. From $\lambda_{j}=\mu-m(\tilde{u}, \tilde{u})$ we get that a critical eigenvector $\tilde{u}$ corresponding to the eigenvalue $\lambda_{j}$ is given by $\tilde{u}=\left(\mu-\lambda_{j}\right)^{1 / 2} e$ with $\mathscr{M}(e)=1$, and (i) holds.

Evaluation of $\mathscr{G}(\cdot, \mu)$ at $\tilde{u}$ gives

$$
\mathscr{G}(\tilde{u}, \mu)=-\frac{1}{2}\left(\mu-\lambda_{j}\right)^{2}
$$

as the associated critical value of $\mathscr{G}(\cdot, \mu)$. This will be minimized when $j=1$, and then the minimizers of $\mathscr{G}(\cdot, \mu)$ are $\tilde{u}=\left(\mu-\lambda_{1}\right)^{1 / 2} e$ with $e$ an eigenvector corresponding to the first eigenvalue and $\mathscr{M}(e)=1$.

That is, for given $\mu$, the minimum value of this problem is a function of $\lambda_{1}$, and the minimizers are eigenvectors of $(a, m)$ associated with $\lambda_{1}$.

Given $\mu \in(0, \infty)$, Theorems 4.2 and 4.3 show that the unconstrained variational principle $\left(\mathcal{P}_{\mu}\right)$ provides upper and lower bounds on $\lambda_{1}$ as described in the next result.

Corollary 4.4. Assume (A1)-(A3) hold and $\mathscr{G}(\cdot, \mu)$ is defined by (4.1).

(i) If $\alpha(\mu)=0$, then $\mu \leq \lambda_{1}$.

(ii) If there exists $\widetilde{w} \in V$ with $\mathscr{G}(\widetilde{w}, \mu)<0$, then

$$
\lambda_{1}=\inf _{\mathscr{G}(u, \mu)<0}[\mu-\sqrt{-2 \mathscr{G}(u, \mu)}] \leq \mu-\sqrt{-2 \mathscr{G}(\widetilde{w}, \mu)} .
$$


Proof. The first assertion follows from Theorem 4.3. When $\widetilde{w} \in V$ has $\mathscr{G}(\widetilde{w}, \mu)<0$, then the preceding theorem yields

$$
-\frac{1}{2}\left(\mu-\lambda_{1}\right)^{2}=\alpha(\mu) \leq \mathscr{G}(\widetilde{w}, \mu)
$$

so the second part holds on rearrangement.

Given a distinct eigenvalue $\tilde{\lambda}_{j}$ of $(a, m)$ in the interval $(0, \mu)$, let $E_{j}$ be the eigenspace corresponding to $\tilde{\lambda}_{j}$, $m_{j}$ be the dimension of $E_{j}$ and $C\left(\tilde{\lambda}_{j} ; \mu\right)$ be the subset in $E_{j}$ defined by

$$
C\left(\tilde{\lambda}_{j} ; \mu\right):=\left\{u \in E_{j}:\|u\|_{m}^{2}=\mu-\tilde{\lambda}_{j}\right\}
$$

where $\|u\|_{m}^{2}:=\mathscr{M}(u)$. These sets may be characterized as follows.

Theorem 4.5. Assume (A1)-(A3) hold, $\mu>\tilde{\lambda}_{j}$ and the set $C\left(\tilde{\lambda}_{j} ; \mu\right)$ is defined as above.

(i) If $\tilde{\lambda}_{j}$ is a simple eigenvalue of $(a, m)$, then $C\left(\tilde{\lambda}_{j} ; \mu\right)$ consists of exactly two points.

(ii) If $\tilde{\lambda}_{j}$ is an eigenvalue of multiplicity $m_{j} \geq 2$, then $C\left(\tilde{\lambda}_{j} ; \mu\right)$ is diffeomorphic to a sphere of dimension $m_{j}-1$.

(iii) If $\tilde{u}_{j} \in C\left(\tilde{\lambda}_{j} ; \mu\right)$ and $\tilde{u}_{k} \in C\left(\tilde{\lambda}_{k} ; \mu\right)$, with $k \neq j$, then

$$
\mathscr{M}\left(\tilde{u}_{j}-\tilde{u}_{k}\right)=2 \mu-\left(\tilde{\lambda}_{j}+\tilde{\lambda}_{k}\right) .
$$

Proof. If the eigenvalue $\tilde{\lambda}_{j} \in(0, \mu)$ is simple, then

$$
C\left(\tilde{\lambda}_{j} ; \mu\right)=\left\{ \pm\left(\mu-\tilde{\lambda}_{j}\right)^{1 / 2} \tilde{e}_{j}\right\}
$$

where $\tilde{e}_{j}$ is an eigenvector of the pair $(a, m)$ corresponding to $\tilde{\lambda}_{j}$ with $\left\|\tilde{e}_{j}\right\|_{m}=1$.

If $\tilde{\lambda}_{j} \in(0, \mu)$ is an eigenvalue of $(a, m)$ of multiplicity $m_{j} \geq 2$, let $\left\{\tilde{e}_{j}, \tilde{e}_{j+1}, \ldots, \tilde{e}_{j+m_{j}-1}\right\}$ be an $m$-orthonormal basis of the eigenspace $E_{j}$. A calculation then shows that

$$
C\left(\tilde{\lambda}_{j} ; \mu\right)=\left\{u \in V: u=\sum_{k=1}^{m_{j}} c_{k} \tilde{e}_{j+k-1} \text { with } \sum_{k=1}^{m_{j}} c_{k}^{2}=\mu-\tilde{\lambda}_{j}\right\},
$$

which is diffeomorphic to to an $\left(m_{j}-1\right)$-dimensional sphere.

To obtain (iii), we see that for $\tilde{u}_{j} \in C\left(\tilde{\lambda}_{j} ; \mu\right)$ and $\tilde{u}_{k} \in C\left(\tilde{\lambda}_{k} ; \mu\right)$,

$$
\tilde{u}_{j}=\left(\mu-\tilde{\lambda}_{j}\right)^{1 / 2} \tilde{e}_{j} \quad \text { and } \quad \tilde{u}_{k}=\left(\mu-\tilde{\lambda}_{k}\right)^{1 / 2} \tilde{e}_{k}
$$

for some $m$-normalized eigenvectors $\tilde{e}_{j}, \tilde{e}_{k}$ of $(a, m)$ corresponding to eigenvalues $\tilde{\lambda}_{j}, \tilde{\lambda}_{k}$. Then (4.8) follows upon substitution.

In view of this result, we can describe the set of all non-zero critical points of $\mathscr{G}(\cdot, \mu)$ to be the disjoint union of finitely many finite-dimensional spheres $C\left(\tilde{\lambda}_{j} ; \mu\right)$, the spheres being pairwise $m$-orthogonal. Let

$$
C(\mu):=\cup_{\tilde{\lambda}_{j}<\mu} C\left(\tilde{\lambda}_{j} ; \mu\right) .
$$

Corollary 4.6. Under the conditions of the preceding theorem, $C(\mu)$ is bounded and consists of finitely many closed connected components and finitely many points. If each eigenvalue of $(a, m)$ is simple then $C(\mu)$ consists of $2 M$ points where $M$ is the number of eigenvalues of $(a, m)$ less than $\mu$. 


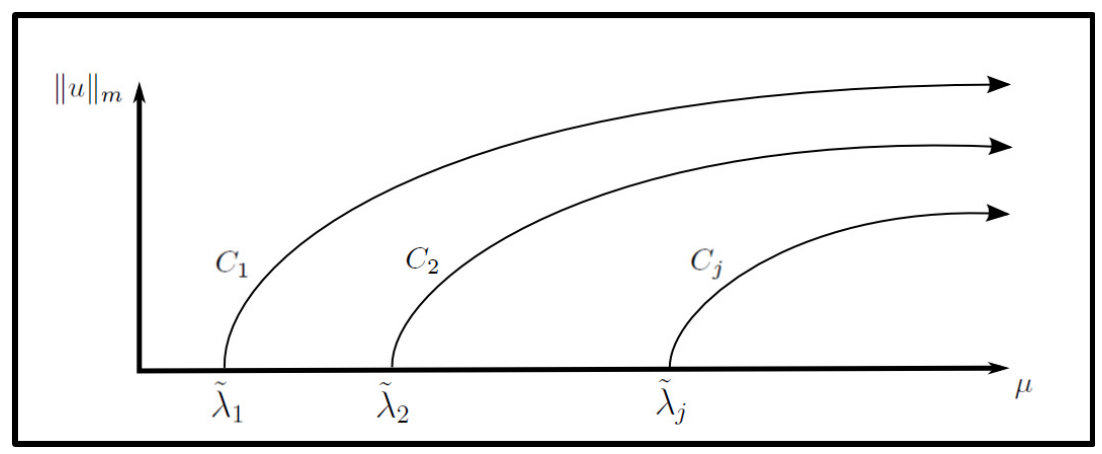

Figure 1. Bifurcation diagram for $\mathscr{G}(\cdot, \mu)$.

When the parameter $\mu$ increases, the number of critical points may increase. The above is a schematic bifurcation diagram for the critical points of $\mathscr{G}(\cdot, \mu)$.

In Figure 1,

$$
C_{j}=\left\{\left(\tilde{\lambda}_{j}+s, s^{1 / 2} e\right): s \geq 0, e \in E_{j},\|e\|_{m}=1\right\}
$$

is the bifurcation branch of the set of critical points corresponding to $\tilde{\lambda}_{j}$, the $j$ th distinct eigenvalue of $(a, m)$. The sphere $C\left(\tilde{\lambda}_{j} ; \mu\right)$ is thought of as a point on the branch $C_{j}$.

From Theorem 4.2 we see that 0 is a critical point for any value of $\mu$, and it is the unique critical point when $\mu \leq \lambda_{1}$. As $\mu$ increases through an eigenvalue $\tilde{\lambda}_{j}$, a new sphere $C\left(\tilde{\lambda}_{j} ; \mu\right)$ of critical points emanates from the origin and moves along the branch $C_{j}$. That is, each sphere of non-zero critical points, centered at the origin, persists and expands in $V$ as the parameter $\mu$ increases, without any further bifurcations.

\section{TyPES AND MORSE INDICES OF CRITICAL POINTS}

This section describes the degeneracy and indices of the critical points of the functional $\mathscr{G}(\cdot, \mu)$. We show that a critical point is associated with a simple eigenvalue of $(a, m)$ if and only if the critical point is non-degenerate and then the Morse index of the critical point is related to the number of eigenvalues that are less than this eigenvalue. The results are analogous to the famous Courant-Fischer-Weyl min-max results for constrained variational problems associated with the use of Rayleigh quotients.

As in the previous section, the distinct eigenvalues of $(a, m)$ will be denoted $\tilde{\lambda}_{j}$ so that $0<\tilde{\lambda}_{1}<\tilde{\lambda}_{2}<\ldots$ and $m_{j}$ is the multiplicity of $\tilde{\lambda}_{j}$ as an eigenvalue of $(a, m)$. Thus $\lambda_{1}=\tilde{\lambda}_{1}$ but $\lambda_{2}<\tilde{\lambda}_{2}$ when $\lambda_{1}$ has multiplicity $m_{1} \geq 2$. The set of distinct eigenvalues of $(a, m)$ will be called the spectrum of $(a, m)$ and denoted $\sigma(a, m)$.

The expression for the Hessian form of $\mathscr{G}(\cdot, \mu)$ described in Theorem 4.1 will be used to determine the type of critical points of $\mathscr{G}(\cdot, \mu)$. First, the index of the critical point at the origin may be found by using $\delta^{2} \mathscr{G}(0, \mu)(v, w)=2 a(v, w)-2 \mu m(v, w)$. Denote this bilinear form by $h_{0}(\mu)(v, w)$. Thus we have the following result which implies that 0 is not a local minimizer of $\mathscr{G}(\cdot, \mu)$ when $\mu>\lambda_{1}$.

Theorem 5.1. Assume (A1)-(A4) hold, and $\mathscr{G}$ is defined by (4.1). Then 0 is a non-degenerate critical point of $\mathscr{G}(\cdot, \mu)$ if and only if $\mu$ is not an eigenvalue of $(a, m)$. The Morse index $i(0 ; \mu)$ of 0 is zero when $\mu<\lambda_{1}$ and $i(0 ; \mu)=\sum_{\tilde{\lambda}_{k}<\mu} m_{k}$ when $\mu>\lambda_{1}$ and $\mu \notin \sigma(a, m)$. When 0 is a degenerate critical point of $\mathscr{G}\left(\cdot, \tilde{\lambda}_{j}\right)$ then its null index is $i_{0}\left(0 ; \tilde{\lambda}_{j}\right)=m_{j}$.

Proof. From Theorem 2.1, there is a set of eigenvectors $\mathcal{E}:=\left\{e_{j}: j \geq 1\right\}$ corresponding to strictly positive eigenvalues $\lambda_{1} \leq \lambda_{2} \leq \ldots$ of $(a, m)$ that is an $m$-orthonormal basis of $V$.

Taking $v=e_{j}$ in the expression for the second variation at 0 gives

$$
h_{0}(\mu)\left(e_{j}, w\right)=2\left(\lambda_{j}-\mu\right) m\left(e_{j}, w\right) \quad \text { for all } \quad w \in V .
$$


so that $\mathcal{E}$ is an $m$-orthonormal set of eigenvectors for the pair $\left(h_{0}(\mu), m\right)$ with eigenvalues $2\left(\lambda_{j}-\mu\right)$. Thus the first result follows.

Now

$$
h_{0}(\mu)(v, v) \geq 2 k_{0}\|v\|_{V}^{2}-2 \mu m(v, v) \quad \text { for all } \quad v \in V
$$

so (M1), (M2) hold for $\left(h_{0}(\mu), m\right)$. Then Theorem 3.1 and (5.1) imply the second statement as there are no negative eigenvalues of $\left(h_{0}(\mu), m\right)$ when $\mu<\lambda_{1}$. Implied also is that the Morse index of 0 will be the number of eigenvalues $\lambda_{j}$ of $(a, m)$ with $\lambda_{j}<\mu$, counting multiplicities, when $\mu>\lambda_{1}$ and $\mu \notin \sigma(a, m)$. Thus the formula for this Morse index $i(0 ; \mu)$ holds. The last statement is direct.

When $\tilde{u}_{j}$ is a non-zero critical point of $\mathscr{G}(\cdot, \mu)$ associated with the eigenvalue $\tilde{\lambda}_{j}$, then the Hessian at $\tilde{u}_{j}$ is

$$
\delta^{2} \mathscr{G}\left(\tilde{u}_{j}, \mu\right)(v, w)=2 a(v, w)-2 \tilde{\lambda}_{j} m(v, w)+4\left(\mu-\tilde{\lambda}_{j}\right) m(e, v) m(e, w)
$$

since $\tilde{u}_{j}=\left(\mu-\tilde{\lambda}_{j}\right)^{1 / 2} e$ with $e$ an $m$-normalized eigenvector corresponding to $\tilde{\lambda}_{j} \in(0, \mu)$. Denote this Hessian bilinear form by $h_{j}(\mu)$. Note that for all such $\tilde{u}_{j}$ and $\mu$, this bilinear form satisfies

$$
h_{j}(\mu)(v, v) \geq 2 k_{0}\|v\|_{V}^{2}-2 \tilde{\lambda}_{j} m(v, v),
$$

so that the pair $\left(h_{j}(\mu), m\right)$ satisfies (M1), (M2) with $h_{j}(\mu)$ in place of $b$.

The following result describes conditions for such critical points to be non-degenerate and what their Morse indices may be.

Theorem 5.2. Assume (A1)-(A4) hold, $\mu>\tilde{\lambda}_{j}$, and $\mathscr{G}(\cdot, \mu)$ is defined by (4.1). Suppose $\tilde{u}_{j}$ is a non-zero critical point of $\mathscr{G}(\cdot, \mu)$ associated with the eigenvalue $\tilde{\lambda}_{j}$ of $(a, m)$. Then $\tilde{u}_{j}$ is a non-degenerate critical point of $\mathscr{G}(\cdot, \mu)$ if and only if $\tilde{\lambda}_{j}$ is a simple eigenvalue of $(a, m)$. In this case the Morse index of $\tilde{u}_{j}$ is $i\left(\tilde{u}_{j} ; \mu\right)=$ $\sum_{k=1}^{j-1} m_{k}$. If $\tilde{\lambda}_{j}$ has multiplicity $m_{j} \geq 2$ then $\tilde{u}_{j}$ is a degenerate critical point of $\mathscr{G}(\cdot, \mu)$ that has null index $i_{0}\left(\tilde{u}_{j} ; \mu\right)=m_{j}-1$.

Proof. Let $\mathcal{E}$ and $\lambda_{1} \leq \lambda_{2} \leq \ldots \leq \lambda_{j} \leq \ldots$ be as in the previous theorem. Without loss of generality suppose $e=e_{k}$ for some $e_{k} \in \mathcal{E} \cap E_{\tilde{\lambda}_{j}}$. Taking $v=e_{i}$ in (5.2) yields

$$
h_{j}(\mu)\left(e_{i}, w\right)=\left\{\begin{array}{lll}
4\left(\mu-\tilde{\lambda}_{j}\right) m\left(e_{i}, w\right) & \text { if } & i=k, \\
2\left(\lambda_{i}-\tilde{\lambda}_{j}\right) m\left(e_{i}, w\right) & \text { if } & i \neq k
\end{array}\right.
$$

This shows that $\mathcal{E}$ is an $m$-orthonormal set of eigenvectors for the pair $\left(h_{j}(\mu), m\right)$ corresponding to eigenvalues $4\left(\mu-\tilde{\lambda}_{j}\right)$, and $2\left(\lambda_{i}-\tilde{\lambda}_{j}\right)$ for $i \neq k$.

Suppose $h_{j}(\mu)$ is non-degenerate. If the multiplicity of $\tilde{\lambda}_{j}$ is $m_{j} \geq 2$, then (5.3) implies that, for some $e_{i} \in \mathcal{E} \cap E_{\tilde{\lambda}_{j}}$,

$$
h_{j}(\mu)\left(e_{i}, w\right)=0 \text { for all } w \in V .
$$

This contradicts the non-degeneracy of the Hessian $h_{j}(\mu)$ of $\mathscr{G}(\cdot, \mu)$ at $\tilde{u}_{j}$, so $\tilde{\lambda}_{j}$ must be a simple eigenvalue of $(a, m)$.

On the other hand, if $h_{j}(\mu)$ is degenerate, there is a nonzero $v \in V$ satisfying

$$
h_{j}(\mu)(v, w)=0 \text { for all } w \in V .
$$

By (5.3), this implies $\lambda_{i}=\tilde{\lambda}_{j}$ for some $i \neq k$, so that $\tilde{\lambda}_{j}$ is not simple and the first assertion holds.

When $\tilde{\lambda}_{j}$ is simple, Theorem 3.1 implies that the Morse index $i\left(\tilde{u}_{j} ; \mu\right)$ of $h_{j}(\mu)$ is equal to the number of negative eigenvalues $2\left(\lambda_{i}-\tilde{\lambda}_{j}\right)$ of the pair $\left(h_{j}(\mu), m\right)$, counting multiplicities. This is the number of eigenvalues $\lambda_{i}$ that are strictly less than $\tilde{\lambda}_{j}$, so the second assertion follows.

The null index $i_{0}\left(\tilde{u}_{j} ; \mu\right)$ of a degenerate critical point $\tilde{u}_{j}$ of $\mathscr{G}(\cdot, \mu)$ is equal to the multiplicity of 0 as an eigenvalue of the pair $\left(h_{j}(\mu), m\right)$. Thus the last sentence holds. 
Theorem 5.2 provides additional information on the schematic bifurcation diagram in Figure 1. From Theorem 4.3 , when $\mu>\tilde{\lambda}_{1}$, the minimizers of $\mathscr{G}(\cdot, \mu)$ are critical points $\tilde{u}_{1}$ with Morse index zero and null index $i_{0}\left(\tilde{u}_{1} ; \mu\right)=m_{1}-1$. These indices are constant when $C\left(\tilde{\lambda}_{1} ; \mu\right)$ moves along the branch $C_{1}$. When $\mu>\tilde{\lambda}_{j}$, where $j \geq 2$ and $\tilde{\lambda}_{j}$ is the $j$ th distinct smallest eigenvalue of $(a, m)$, the points $\tilde{u}_{j}$ on the sphere $C\left(\tilde{\lambda}_{j} ; \mu\right)$ are saddle points of $\mathscr{G}(\cdot, \mu)$ with Morse and null index, respectively,

$$
i\left(\tilde{u}_{j} ; \mu\right)=\sum_{k=1}^{j-1} m_{k} \quad \text { and } \quad i_{0}\left(\tilde{u}_{j} ; \mu\right)=m_{j}-1 .
$$

These indices are constant as $C\left(\tilde{\lambda}_{j} ; \mu\right)$ moves along the branch $C_{j}$.

For the trivial branch, Theorem 5.1 says that as $\mu$ passes through $\tilde{\lambda}_{j}$, an $\left(m_{j}-1\right)$-dimensional sphere bifurcates from the origin. In particular the Morse index of 0 increases by $m_{j}$ when $\mu$ passes through $\tilde{\lambda}_{j}$.

\section{Penalization of the functional $\mathscr{G}(\cdot, \mu)$}

The preceding analysis shows that when $\mu>\lambda_{1}$, the global minimizer of the functional $\mathscr{G}(\cdot, \mu)$ is attained at vectors in the eigenspace $E_{1}$ corresponding to the least eigenvalue $\lambda_{1}$. Here penalizations of the functional $\mathscr{G}$ in a manner that has long been used by numerical analysts will be used to provide unconstrained variational principles for the second eigenvalue and associated eigenvectors.

Suppose that we know an $m$-normalized eigenvector $e_{1}$ corresponding to the first eigenvalue $\lambda_{1}$ of $(a, m)$. Define a functional $\mathscr{G}_{\tau}: V \times(0, \infty) \rightarrow \mathbb{R}$ by

$$
\mathscr{G}_{\tau}(u, \mu):=\mathscr{G}(u, \mu)+\tau m\left(e_{1}, u\right)^{2} .
$$

Here $\tau \geq 0$ is often called a penalty parameter.

Consider the unconstrained variational problem $\left(\mathcal{P}_{\mu, \tau}\right)$ of minimizing $\mathscr{G}_{\tau}(\cdot, \mu)$ on $V$ and evaluating

$$
\alpha(\mu, \tau):=\inf _{u \in V} \mathscr{G}_{\tau}(u, \mu)
$$

The variations of the functional $\mathscr{G}_{\tau}(\cdot, \mu)$ at $u \in V$ are easily found to be

$$
\begin{aligned}
\delta \mathscr{G}_{\tau}(u ; v ; \mu) & =2\left\{a(u, v)+[m(u, u)-\mu] m(u, v)+\tau m\left(e_{1}, u\right) m\left(e_{1}, v\right)\right\} \\
\delta^{2} \mathscr{G}_{\tau}(u ; v, w ; \mu) & =\delta^{2} \mathscr{G}(u ; v, w ; \mu)+2 \tau m\left(e_{1}, v\right) m\left(e_{1}, w\right) .
\end{aligned}
$$

From (6.3), a vector $u \in V$ is a critical point of $\mathscr{G}_{\tau}(\cdot, \mu)$ provided

$$
a(u, v)=[\mu-m(u, u)] m(u, v)-\tau m\left(e_{1}, u\right) m\left(e_{1}, v\right) \text { for all } v \in V .
$$

Note that a critical point $u$ of $\mathscr{G}(\cdot, \mu)$ remains a critical point of $\mathscr{G}_{\tau}(\cdot, \mu)$ for all $\tau>0$ provided $m\left(e_{1}, u\right)=0$. However their Morse indices may change. Also the zero vector is still a critical point of this penalized functional but it is now a minimizer for a larger range of values of $\mu$ - at least when the least eigenvalue is simple. Let $\mu_{c}:=\min \left\{\lambda_{1}+2 \tau, \lambda_{2}\right\}$, and recall the $\lambda_{j}$ 's take multiplicity into account, whereas the $\tilde{\lambda}_{j}$ 's do not.

Theorem 6.1. Assume (A1)-(A3) hold, the sets $C\left(\tilde{\lambda}_{j} ; \mu\right)$ are given by (4.7), and $\mathscr{G}_{\tau}$ is defined by (6.1).

(i) If $0<\mu<\mu_{c}$ and $\lambda_{1}$ is a simple eigenvalue of $(a, m)$, then 0 minimizes $\mathscr{G}_{\tau}(\cdot, \mu)$ on $V$.

(ii) If $\mu>\mu_{c}, \tau>\lambda_{2}-\lambda_{1}$ and $\lambda_{1}$ is a simple eigenvalue of $(a, m)$, then the minimizers of $\mathscr{G}_{\tau}(\cdot, \mu)$ on $V$ are the vectors in $C\left(\tilde{\lambda}_{2} ; \mu\right)$ and

$$
\alpha(\mu, \tau)=-\frac{1}{2}\left(\mu-\tilde{\lambda}_{2}\right)^{2}>\alpha(\mu) .
$$


(iii) If $\mu>\mu_{c}$ and $\lambda_{1}$ is an eigenvalue of multiplicity $m_{1} \geq 2$, then the minimizers of $\mathscr{G}_{\tau}(\cdot, \mu)$ on $V$ are the vectors $u$ in $C\left(\tilde{\lambda}_{1} ; \mu\right)$ that also satisfy $m\left(e_{1}, u\right)=0$. In this case $\alpha(\mu, \tau)=\alpha(\mu)$.

Proof. Note that as the penalty parameter $\tau$ is positive, we have

$$
\mathscr{G}_{\tau}(u, \mu) \geq \mathscr{G}(u, \mu) \text { for all }(u, \mu) \in V \times(0, \infty) .
$$

Evaluating $\mathscr{G}_{\tau}(\cdot, \mu)$ at a critical point $\tilde{u}_{j}=\left(\mu-\tilde{\lambda}_{j}\right)^{1 / 2} e$ of $\mathscr{G}(\cdot, \mu)$, with $e$ an $m$-normalized eigenvector of $(a, m)$ in $E_{j}$ gives $\mathscr{G}_{\tau}\left(\tilde{u}_{j}, \mu\right)=-\frac{1}{2}\left(\mu-\tilde{\lambda}_{j}\right)^{2}+\tau\left(\mu-\tilde{\lambda}_{j}\right) m\left(e_{1}, e\right)^{2}$ which becomes

$$
\mathscr{G}_{\tau}\left(\tilde{u}_{j}, \mu\right)=\left\{\begin{array}{lll}
-\frac{1}{2}\left(\mu-\lambda_{1}\right)^{2}+\tau\left(\mu-\lambda_{1}\right) & \text { if } & m\left(e_{1}, e\right)=1, \\
-\frac{1}{2}\left(\mu-\tilde{\lambda}_{j}\right)^{2} & \text { if } & m\left(e_{1}, e\right)=0 .
\end{array}\right.
$$

When $\mu<\mu_{c}$, the only possible critical points $\tilde{u}_{j}$ of $\mathscr{G}(\cdot, \mu)$ are the points $\tilde{u}_{1}$ in $E_{1}$.

From (6.6), we see that when $\lambda_{1}$ is simple, the corresponding critical value of $\mathscr{G}_{\tau}(\cdot, \mu)$ at $\tilde{u}_{1}$ satisfies

$$
\mathscr{G}_{\tau}\left(u_{1}, \mu\right)=-\frac{1}{2}\left(\mu-\lambda_{1}\right)^{2}+\tau\left(\mu-\lambda_{1}\right)>0
$$

as $\mu<\lambda_{1}+2 \tau$, so that $\alpha(\mu, \tau)=0$ and (i) holds.

The inequalities $\mu>\mu_{c}, \tau>\lambda_{2}-\lambda_{1}$ imply $\mu>\lambda_{2}$, when $\lambda_{1}$ is simple. Thus we have critical points $\tilde{u}_{1}, \tilde{u}_{2}$ of $\mathscr{G}(\cdot, \mu)$, and consequently of $\mathscr{G}_{\tau}(\cdot, \mu)$, in this case. These conditions also imply

$$
\mathscr{G}_{\tau}\left(\tilde{u}_{2}, \mu\right)=-\frac{1}{2}\left(\mu-\lambda_{2}\right)^{2}<-\frac{1}{2}\left(\mu-\lambda_{1}\right)^{2}+\tau\left(\mu-\lambda_{1}\right)=\mathscr{G}_{\tau}\left(\tilde{u}_{1}, \mu\right)
$$

from (6.6), so that the vectors $\tilde{u}_{2}$ in $C\left(\tilde{\lambda}_{2} ; \mu\right)$ are minimizers of $\mathscr{G}_{\tau}(\cdot, \mu)$, and (ii) holds.

When $\mu>\mu_{c}$ and $\lambda_{1}$ has multiplicity $m_{1} \geq 2$, from (6.6) we see that the corresponding critical value of $\mathscr{G}_{\tau}(\cdot, \mu)$ at any critical point $u_{1}=\left(\mu-\lambda_{1}\right)^{1 / 2} e$ of $\mathscr{G}(\cdot, \mu)$ with $m\left(e_{1}, u_{1}\right)=0$, satisfies

$$
\mathscr{G}_{\tau}\left(u_{1}, \mu\right)=-\frac{1}{2}\left(\mu-\lambda_{1}\right)^{2}<-\frac{1}{2}\left(\mu-\tilde{\lambda}_{j}\right)^{2}=\mathscr{G}_{\tau}\left(u_{j}, \mu\right)
$$

for any critical point $u_{j}$ in $C\left(\tilde{\lambda}_{j} ; \mu\right), j \geq 2$. Also from (6.6)

$$
\mathscr{G}_{\tau}\left(u_{1}, \mu\right)<-\frac{1}{2}\left(\mu-\lambda_{1}\right)^{2}+\tau\left(\mu-\lambda_{1}\right)
$$

for any value of $\tau$, so that (iii) follows.

It is worth noting that, when the least eigenvalue is simple, the difference between the value of the penalized problem $\mathcal{P}_{\mu, \tau}$ and that of $\mathcal{P}_{\mu}$ is a function of the spectral gap $d:=\lambda_{2}-\lambda_{1}$ between the first two eigenvalues of $(a, m)$.

When $\lambda_{1}$ is a simple eigenvalue of $(a, m)$ and $\tau>\lambda_{2}-\lambda_{1}, \mu>\mu_{c}$, then the minimizers of $\mathscr{G}_{\tau}(\cdot, \mu)$ are eigenvectors of $(a, m)$ corresponding to the eigenvalue $\lambda_{2}$. That is, this penalized functional provides an unconstrained variational problem whose minimizers yield the second eigenvalue and associated eigenvectors.

When we know a subspace $V_{1}$ that is spanned by a finite number of eigenvectors of $(a, m)$, there is a penalized functional of this type with the property that when $\mu$ and $\tau$ are sufficiently large, the minimizers of the functional will be eigenvectors of $(a, m)$ that are $m$-orthogonal to $V_{1}$. 


\section{INDEFINITE ELLIPTIC EIGENPROBLEMS}

The preceding sections have treated the eigenproblem (2.1) subject to positivity of the functional $\mathscr{M}$ associated with $m$. Much of the analysis extends to the case where $\mathscr{M}$ can take both positive and negative values. In this section we typically require that $m$ satisfies:

(A5) There exist $v_{1}, v_{2} \in V$ such that $\mathscr{M}\left(v_{1}\right)<0<\mathscr{M}\left(v_{2}\right)$.

An eigenproblem for $(a, m)$ where $m$ satisfies (A2) and (A5) will be called an indefinite eigenproblem. The monograph of Belgacem [7] provides a nice description of many results about such problems.

Results in [6] show that when (A1), (A2) and (A5) hold then there are sets of positive and negative eigenvalues of $(a, m)$. Let $\lambda_{1}^{+}$be the least strictly positive eigenvalue of $(a, m)$ and $\lambda_{1}^{-}$be the strictly negative eigenvalue of $(a, m)$ closest to 0 . These exist from Theorem 3.1 of [6].

Consider the problem of minimizing $\mathscr{G}(\cdot, \mu)$ defined by (4.1) and evaluating $\alpha(\mu)$ defined by (4.2). As Theorem 4.1 only assumes (A1) and (A2), it remains valid when (A5) also holds. Theorems 4.2 and 4.3 change to the following.

Theorem 7.1. Assume (A1), (A2), and (A5) hold, and $\mathscr{G}$ is defined by (4.1). Let $\lambda_{1}^{+}$be the least positive eigenvalue of $(a, m)$.

(i) If $0<\mu \leq \lambda_{1}^{+}$, then 0 is the unique critical point of $\mathscr{G}(\cdot, \mu)$ on $V$ and $\alpha(\mu)=0$.

(ii) If $\mu>\lambda_{1}^{+}$, then the minimizers of $\mathscr{G}(\cdot, \mu)$ on $V$ are $\left(\mu-\lambda_{1}^{+}\right)^{1 / 2} e$, where $e$ is an $m$-normalized eigenvector of $(a, m)$ corresponding to $\lambda_{1}^{+}$, and $2 \alpha(\mu)=-\left(\mu-\lambda_{1}^{+}\right)^{2}$.

Proof. As in the proofs of Theorems 4.2 and 4.3 we see that a critical point $u$ of $\mathscr{G}(\cdot, \mu)$ satisfies

$$
a(u, v)=[\mu-m(u, u)] m(u, v) \text { for all } v \in V .
$$

Thus 0 is always a critical point, and non-zero critical points $u$ are eigenvectors corresponding to eigenvalues $\lambda=\mu-m(u, u)$. By the coercivity of $a(\cdot, \cdot)$, the eigenvalues of $(a, m)$ are non-zero. If $\lambda=\lambda^{+}$is a strictly positive eigenvalue of $(a, m)$, then put $v=u$ in $(7.1)$ to find

$$
a(u, u)=[\mu-m(u, u)] m(u, u)=\lambda^{+}\left(\mu-\lambda^{+}\right)
$$

which implies $\lambda^{+}\left(\mu-\lambda^{+}\right)>0$ as $a(\cdot, \cdot)$ is coercive on $V$. This shows $\lambda^{+}<\mu$.

From (7.1), we also see that if $u$ is an eigenvector associated with a positive eigenvalue $\lambda^{+}$of $(a, m)$, then $m(u, u)>0$.

If $\lambda=\lambda^{-}$is a strictly negative eigenvalue of $(a, m)$, then substituting a corresponding eigenvector $u$ for $v$ in $(7.1)$ gives

$$
\lambda^{-}\left(\mu-\lambda^{-}\right)>0,
$$

again, by the coercivity of $a(\cdot, \cdot)$. This implies $\mu<\lambda^{-}$, a contradiction to $\mu$ being positive. Therefore, the only possible non-zero critical points of $\mathscr{G}(\cdot, \mu)$ are eigenvectors of $(a, m)$ corresponding to eigenvalues $\lambda_{j}$ belonging to the interval $(0, \mu)$.

As in Theorems 4.2 and 4.3 , minimizers of $\mathscr{G}(\cdot, \mu)$ exist and are critical points. Therefore, when $\mu \in\left(0, \lambda_{1}^{+}\right)$, we conclude from the above work that 0 is the only critical point of $\mathscr{G}(\cdot, \mu)$ with value zero, so (i) holds. When $\mu>\lambda_{1}^{+}$, the non-zero critical points of $\mathscr{G}(\cdot, \mu)$ are

$$
\left(\mu-\tilde{\lambda}_{j}^{+}\right)^{1 / 2} \tilde{e}_{j}^{+}
$$

with $\tilde{e}_{j}^{+}$an $m$-normalized eigenvector corresponding to the $j$ th distinct, smallest, strictly positive eigenvalue ${\tilde{\lambda_{j}}}^{+}$of $(a, m)$ less than $\mu$. By considering the corresponding critical values, (ii) follows. 
When $\mathscr{M}$ satisfies (A5), then the negative eigenvalues $\lambda_{j}^{-}$of $(a, m)$ are precisely the positive eigenvalues of $(a,-m)$. Thus negative eigenvalues of $(a, m)$ may be found by considering the problem of minimizing $\mathscr{G}_{-}(\cdot, \mu)$, with $\mu>0$, defined by

$$
\mathscr{G}_{-}(u, \mu):=\mathscr{A}(u)+\mu \mathscr{M}(u)+\frac{1}{2} \mathscr{M}(u)^{2} .
$$

Let $\alpha_{-}(\mu):=\inf _{u \in V} \mathscr{G}_{-}(u, \mu)$. The following result about the critical points and minimizers of $\mathscr{G}_{-}(\cdot, \mu)$ now follows from this theorem.

Corollary 7.2. Assume (A1), (A2), and (A5) hold, and $\mathscr{G}_{-}$is defined by (7.2). Let $\lambda_{1}^{-}$be the largest negative eigenvalue of $(a, m)$.

(i) If $0<\mu \leq-\lambda_{1}^{-}$, then 0 is the unique critical point of $\mathscr{G}_{-}(\cdot, \mu)$ on $V$ and $\alpha_{-}(\mu)=0$.

(ii) If $\mu>-\lambda_{1}^{-}$, then the minimizers of $\mathscr{G}_{-}(\cdot, \mu)$ on $V$ are $\left(\mu+\lambda_{1}^{-}\right)^{1 / 2} e$, where $e$ is an eigenvector of $(a, m)$ corresponding to $\lambda_{1}^{-}$satisfying $m(e, e)=-1$, and $2 \alpha(\mu)=-\left(\mu+\lambda_{1}^{-}\right)^{2}$.

The surprising result is that the Morse indices of critical points of $\mathscr{G}(\cdot, \mu)$ and $\mathscr{G}_{-}(\cdot, \mu)$ are related to the ordering of the associated positive and negative eigenvalues of $(a, m)$.

We shall evaluate the Morse and null indices for the critical points for these problems by considering them as critical points of the given functional restricted to a certain special subspace of $V$.

The decomposition

$$
V=V_{+} \oplus_{a} V_{0} \oplus_{a} V_{-}
$$

is given by Corollary 4.5 of [6], when (A1), (A2) and (A5) hold. . Here $\oplus_{a}$ indicates an $a$-orhogonal direct sum, $V_{+}$is the closed subspace of $V$ generated by the eigenvectors $\mathcal{E}_{+}:=\left\{e_{j}^{+}: j \in J_{+}\right\}$associated with strictly positive eigenvalues of $(a, m), V_{-}$is the closed subspace of $V$ generated by the eigenvectors $\mathcal{E}_{-}:=\left\{e_{j}^{-}: j \in J_{-}\right\}$ associated with strictly negative eigenvalues of $(a, m)$, and $V_{0}=N(m)$ is the null space of $m$. Consider the closed subspace

$$
W:=V_{+} \oplus_{a} V_{-}
$$

generated by the eigenvectors of $(a, m)$, and let $\sigma_{+}(a, m):=\left\{\tilde{\lambda}_{j}^{+}: j \in J\right\}$, and $\sigma_{-}(a, m):=\left\{\tilde{\lambda}_{k}^{-}: k \in K\right\}$ be the positive and negative parts of the spectrum $\sigma(a, m)$ of $(a, m)$ consisting, repectively, of the distinct strictly positive and strictly negative eigenvalues of $(a, m)$, so that

$$
\ldots<\tilde{\lambda}_{2}^{-}<\tilde{\lambda}_{1}^{-}<0<\tilde{\lambda}_{1}^{+}<\tilde{\lambda}_{2}^{+}<\ldots
$$

Denoting by $m_{j}^{+}, m_{j}^{-}$the multiplicities of $\tilde{\lambda}_{j}{ }^{+}, \tilde{\lambda}_{j}{ }^{-}$, respectively, Theorems 5.1 and 5.2 now become

Theorem 7.3. Assume (A1), (A2), and (A5) hold, $W$ is given by (7.4) and $\mathscr{G}: W \rightarrow \mathbb{R}$ is defined by (4.1). Then:

(i) 0 is a non-degenerate critical point of $\mathscr{G}(\cdot, \mu): W \rightarrow \mathbb{R}$ if and only if $\mu$ is not a positive eigenvalue of $(a, m)$. When $\mu<\tilde{\lambda}_{1}^{+}$, the Morse index of 0 is $i(0 ; \mu)=0$, and when $\mu>\tilde{\lambda}_{1}^{+}$then $i(0 ; \mu)=\sum_{\tilde{\lambda}_{j}{ }^{+}<\mu} m_{j}^{+}$ if $\mu \notin \sigma_{+}(a, m)$. When 0 is a degenerate critical point of $\mathscr{G}\left(\cdot, \tilde{\lambda}_{j}{ }^{+}\right): W \rightarrow \mathbb{R}$ then its null index is $i_{0}\left(0 ; \tilde{\lambda}_{j}^{+}\right)=m_{j}^{+}$.

(ii) When $\mu>\tilde{\lambda}_{j}^{+}$, then $\tilde{u}_{j}^{+}=\left(\mu-\tilde{\lambda}_{j}^{+}\right)^{1 / 2}$ e, with e an m-normalized eigenvector corresponding to $\tilde{\lambda}_{j}^{+}$, is a non-degenerate critical point of $\mathscr{G}(\cdot, \mu): W \rightarrow \mathbb{R}$ if and only if $\tilde{\lambda}_{j}{ }^{+}$is a simple eigenvalue of $(a, m)$. In this case the Morse index of $\tilde{u}_{j}^{+}$is $i\left(\tilde{u}_{j}^{+} ; \mu\right)=\sum_{k=1}^{j-1} m_{k}^{+}$. If $\tilde{\lambda}_{j}^{+}$has multiplicity $m_{j}^{+} \geq 2$, then $\tilde{u}_{j}^{+}$is a degenerate critical point of $\mathscr{G}(\cdot, \mu): W \rightarrow \mathbb{R}$ that has null index $i_{0}\left(\tilde{u}_{j}^{+} ; \mu\right)=m_{j}^{+}-1$. 
Proof. Define the bilinear form $|m|: W \times W \rightarrow \mathbb{R}$, the absolute value of the bilinear form $m$, as follows. For an eigenvector $e$ corresponding to the eigenvalue $\lambda$ of $(a, m)$, let

$$
|m|(e, e):=\left\{\begin{array}{lll}
m(e, e) & \text { if } \quad \lambda>0 \\
-m(e, e) & \text { if } \quad \lambda<0
\end{array}\right.
$$

and extend to the entire space $W$ using the bilinearity of $m$. We note that for all $w \in W$,

$$
a(e, w)=\lambda m(e, w)= \begin{cases}\lambda|m|(e, w) & \text { if } \quad \lambda>0, \\ |\lambda||m|(e, w) & \text { if } \quad \lambda<0 .\end{cases}
$$

Thus, the eigenvalues of $(a,|m|)$ are the eigenvalues of $(a, m)$ in absolute value. Moreover, the quadratic form $|\mathscr{M}|$ associated to $|m|$ is strictly positive on $W$ and

$$
\mathscr{M}(u) \leq|\mathscr{M}|(u) \text { for all } u \in V .
$$

Considering the Hessian form of $\mathscr{G}(\cdot, \mu)$ at 0 gives

$$
\delta^{2} \mathscr{G}(0, \mu)(v, v)=2 a(v, v)-2 \mu m(v, v) \geq 2 k_{0}\|v\|_{V}^{2}-2 \mu|m|(v, v)
$$

for all $v \in W$. Denote this Hessian bilinear form by $h_{0}(\mu)$. Then the pair $\left(h_{0}(\mu),|m|\right)$ satisfies (M1), (M2) with $\left(h_{0}(\mu),|m|\right)$ in place of $(b, m)$. Taking $e_{j}^{+} \in \mathcal{E}_{+}$for $v$ in $h_{0}(\mu)(v, w)$ yields

$$
h_{0}(\mu)\left(e_{j}^{+}, w\right)=2\left(\lambda_{j}^{+}-\mu\right)|m|\left(e_{j}^{+}, w\right)
$$

and by taking $e_{j}^{-} \in \mathcal{E}_{-}$for $v$ instead we obtain

$$
h_{0}(\mu)\left(e_{j}^{-}, w\right)=2\left(\left|\lambda_{j}^{-}\right|+\mu\right)|m|\left(e_{j}^{-}, w\right) .
$$

A similar argument as in the proof of Theorem 5.1 now yields (i) as $\mathcal{E}_{+} \cup \mathcal{E}_{-}$is an eigenbasis for $W$.

When $\tilde{u}_{j}^{+}=\left(\mu-\tilde{\lambda}_{j}^{+}\right)^{1 / 2} e$ is a critical point of $\mathscr{G}(\cdot, \mu)$ corresponding to $\tilde{\lambda}_{j}^{+} \in(0, \mu)$, the Hessian form of $\mathscr{G}(\cdot, \mu)$ at $\tilde{u}_{j}^{+}$satisfies

$$
\delta^{2} \mathscr{G}\left(\tilde{u}_{j}^{+}, \mu\right)(v, v) \geq 2 a(v, v)-2 \tilde{\lambda}_{j}^{+} m(v, v) \geq 2 k_{0}\|v\|_{V}^{2}-2 \tilde{\lambda}_{j}^{+}|m|(v, v)
$$

for all $v \in W$. Denote this Hessian bilinear form by $h_{j+}(\mu)$. The pair $\left(h_{j+}(\mu),|m|\right)$ thus satisfies (M1), (M2) with $\left(h_{j+}(\mu),|m|\right)$ in place of $(b, m)$. Without loss of generality, suppose $e=e_{k}^{+}$for some $e_{k}^{+} \in \mathcal{E}_{+} \cap E_{\tilde{\lambda}_{j}}+$ Taking $e_{i}^{+} \in \mathcal{E}_{+}$for $v$ in $h_{j+}(\mu)(v, w)$ yields

$$
h_{j+}(\mu)\left(e_{j}^{+}, w\right)= \begin{cases}4\left(\mu-\tilde{\lambda}_{j}^{+}\right)|m|\left(e_{i}^{+}, w\right) & \text { if } \quad i=k \\ 2\left(\lambda_{i}^{+}-\tilde{\lambda}_{j}^{+}\right)|m|\left(e_{i}^{+}, w\right) & \text { if } \quad i \neq k\end{cases}
$$

Taking $v=e_{i}^{-}$instead results in

$$
h_{j+}(\mu)\left(e_{i}^{-}, w\right)=2\left(\left|\lambda_{j}^{-}\right|+\tilde{\lambda}_{j}^{+}\right)|m|\left(e_{i}^{-}, w\right) .
$$

Hence, $\mathcal{E}_{+} \cup \mathcal{E}_{-}$is a set of eigenvectors for $\left(h_{j+}(\mu),|m|\right)$, and a similar argument as in the proof of Theorem 5.2 now yields (ii).

With an appropriate substitution of positive signs with negative signs, one obtains a similar result for the functional $\mathscr{G}_{-}(\cdot, \mu): W \rightarrow \mathbb{R}$ defined by $(7.2)$.

Moreover, higher positive eigenvalues and, respectively, lower negative eigenvalues of $(a, m)$ may be found using penalty methods for $\mathscr{G}(\cdot, \mu)$, respectively $\mathscr{G}_{-}(\cdot, \mu)$, in a manner similar to that of Section 6 . 


\section{UNCONSTRAINED VARIATIONAL PRINCIPLES FOR ROBIN EIGENVALUE PROBLEMS}

The following sections will describe the use of the preceding variational principles for various classes of elliptic eigenproblems. In particular the principles will be applied to some of the problems studied in Auchmuty [6]. In that paper the variational principles for the eigenvalues and eigenvectors are based on the use of constrained optimization characterizations of the eigenvalues of $(a, m)$ - so standard Morse theory is not directly applicable.

First we shall consider variational principles for Robin eigenfunctions and eigenvalues of second-order, divergence form, elliptic equations. There is a (simpler) analogous description for the Dirichlet eigenproblem - but the analysis of Robin eigenproblems illustrates the advantages of using bilinear and quadratic forms compared to the associated linear operators. In particular the inclusion of boundary integrals in the quadratic forms avoids many of the problems that arise in using theories based on closed densely defined linear operators on $L^{2}(\Omega)$ for characterizing the eigenvalues and eigenfunctions.

For all these examples $\langle\cdot, \cdot\rangle$ denotes the usual Euclidean inner product on $\mathbb{R}^{N}$, and $|\cdot|$ the corresponding Euclidean norm. A region $\Omega$ is a non-empty open connected set in $\mathbb{R}^{N}$ and $\sigma, \mathrm{d} \sigma$, respectively represent Hausdorff $(N-1)$-dimensional measure and integration with respect to this measure. All other definitions and notation should be taken to be as in [6] if not given explicitly here. The first requirement on the region $\Omega$ is the following

(B1) $\Omega$ is a bounded region in $\mathbb{R}^{N}$ with boundary $\partial \Omega$ being the union of a finite number of disjoint closed Lipschitz surfaces.

Let $L^{p}(\Omega), L^{q}(\partial \Omega, \mathrm{d} \sigma)$ be the usual real Lebesgue spaces on $\Omega$ and $\partial \Omega$ with norm $\|\cdot\|_{p}$ and $\|\cdot\|_{q, \partial \Omega}$, respectively. Let $H^{1}(\Omega)$ be the real Sobolev space of functions on $\Omega$ with the standard $H^{1}$-inner product

$$
[u, v]_{1}:=\int_{\Omega}[u(x) v(x)+\nabla u(x) \cdot \nabla v(x)] \mathrm{d} x .
$$

This is a Hilbert space and the associated $H^{1}$-norm is denoted $\|\cdot\|_{1,2}$. Take $V$ to be $H^{1}(\Omega)$ in the following.

The region $\Omega$ is said to satisfy the Rellich-Kondrachov theorem provided the imbedding of $H^{1}(\Omega)$ into $L^{p}(\Omega)$ is compact for $1 \leq p<p_{S}$ and $p_{S}=2 N /(N-2)$ when $N \geq 3\left(p_{S}=\infty\right.$ when $\left.N=2\right)$.

The region $\Omega$ is said to satisfy the $L^{2}$-compact trace theorem provided the trace map of $H^{1}(\Omega)$ into $L^{2}(\partial \Omega, \mathrm{d} \sigma)$ is compact. We shall always require that the region satisfy

(B2) $\Omega$ is a region such that (B1), the Rellich-Kondrachov theorem, and the $L^{2}$-compact trace theorem hold.

Consider the problem of finding non-trivial solutions $(\lambda, u)$ of

$$
L u(x):=-\operatorname{div}(A(x) \nabla u(x))+c(x) u(x)=\lambda m_{0}(x) u(x) \quad \text { on } \Omega
$$

subject to

$$
(A(x) \nabla u(x)) \cdot \nu(x)+b(x) u(x)=0 \quad \text { on } \partial \Omega,
$$

where $c, m_{0}, b$ are given functions and $A$ a given matrix-valued field.

This is the Neumann eigenproblem for $\left(L, m_{0}\right)$ on $\Omega$ when $b \equiv 0$ on $\partial \Omega$. Otherwise it is the Robin eigenproblem for $\left(L, m_{0}, b\right)$ on $\Omega$.

The weak form of the boundary value problem (8.1) and (8.2) is to find non-trivial solutions $(\lambda, u) \in$ $\mathbb{R} \times H^{1}(\Omega)$ of

$$
\int_{\Omega}[(A \nabla u) \cdot \nabla v+c u v] \mathrm{d} x+\int_{\partial \Omega} b u v \mathrm{~d} \sigma=\lambda \int_{\Omega} m_{0} u v \mathrm{~d} x \text { for all } v \in H^{1}(\Omega) .
$$

The following conditions on these coefficients will be required. 
(B3) $A(x):=\left(a_{i j}(x)\right)$ is a real symmetric matrix whose components are bounded Lebesgue-measurable functions on $\Omega$ and there exist constants $0<k_{2} \leq k_{3}$ such that

$$
k_{2}|\xi|^{2} \leq\langle A(x) \xi, \xi\rangle \leq k_{3}|\xi|^{2} \quad \text { for all } \xi \in \mathbb{R}^{N}, x \in \Omega .
$$

(B4) $c \geq 0$ and $c \in L^{p}(\Omega)$ for some $p \geq N / 2$ when $N \geq 3$, or $p>1$ when $N=2$.

(B5) $b \in L^{\infty}(\partial \Omega)$ with $b \geq 0 \sigma$-a.e. on $\partial \Omega$, and

$$
\int_{\Omega} c \mathrm{~d} x+\int_{\partial \Omega} b \mathrm{~d} \sigma=b_{0}>0
$$

(B6) $m_{0} \in L^{q}(\Omega)$ for some $q>N / 2$ with $m_{0}$ positive on $\Omega$ and $\left\|m_{0}\right\|_{1}>0$.

The associated bilinear forms for this eigenvalue problem are, with $u, v \in H^{1}(\Omega)$,

$$
\begin{aligned}
a(u, v) & :=\int_{\Omega}[(A \nabla u) \cdot \nabla v+c u v] \mathrm{d} x+\int_{\partial \Omega} b u v \mathrm{~d} \sigma \\
m(u, v) & :=\int_{\Omega} m_{0} u v \mathrm{~d} x .
\end{aligned}
$$

When (B2)-(B6) hold, Lemma 7.1 of [6] shows the bilinear form $m$ satisfies (A2), (A3) with $V=H^{1}(\Omega)$. Theorem 7.2 in [6] shows $a(\cdot, \cdot)$ satisfies (A1) and gives the existence of an increasing sequence of strictly positive eigenvalues $\Lambda:=\left\{\lambda_{j}: j \in \mathbb{N}\right\}$, counting multiplicities, and an associated sequence $\mathcal{E}:=\left\{e_{j}: j \in \mathbb{N}\right\}$ of $m$-orthonormal eigenfunctions for the pair $(a, m)$ in (8.6) and (8.7). Thus there is a least strictly positive eigenvalue $\lambda_{1}$ of (8.3), and a corresponding eigenvector.

The parametrized functional, corresponding to $\mathscr{G}$ of $(4.1)$, for this problem is $\mathscr{R}: H^{1}(\Omega) \times(0, \infty) \rightarrow \mathbb{R}$ defined by

$$
\mathscr{R}(u, \mu)=\int_{\Omega}\left[(A \nabla u) \cdot \nabla u+\left(c-\mu m_{0}\right) u^{2}\right] \mathrm{d} x+\int_{\partial \Omega} b u^{2} \mathrm{~d} \sigma+\frac{1}{2}\left[\int_{\Omega} m_{0} u^{2} \mathrm{~d} x\right]^{2} .
$$

Consider the (unconstrained) variational problem $\left(\mathcal{R}_{\mu}\right)$ of minimizing $\mathscr{R}(\cdot, \mu)$ on $H^{1}(\Omega)$ and finding

$$
\alpha(\mu)=\inf _{u \in H^{1}(\Omega)} \mathscr{R}(u, \mu) .
$$

The essential properties of this data and the functional $\mathscr{R}(\cdot, \mu)$ may be summarized as follows.

Lemma 8.1. Assume (B2)-(B6) hold, and $a, m, \mathscr{R}(\cdot, \mu)$ are defined by (8.6) and (8.8), respectively. Then $a(\cdot, \cdot)$ is an equivalent inner product on $H^{1}(\Omega)$,

(i) $\mathscr{R}(\cdot, \mu)$ is continuous, coercive and weakly l.s.c. on $H^{1}(\Omega)$, and,

(ii) $\mathscr{R}(\cdot, \mu)$ is Gâteaux differentiable on $H^{1}(\Omega)$ with first variation at $u$ in the direction $v$ given by

$$
\delta \mathscr{R}(u ; v ; \mu)=2 a(u, v)+2\left[\int_{\Omega} m_{0} u^{2} \mathrm{~d} x-\mu\right] m(u, v)
$$

Proof. As discussed above, $a(\cdot, \cdot)$ satisfies (A1), hence is an equivalent inner product on $H^{1}(\Omega)$ by $(2.3)$. As the bilinear forms $a, m$ satisfy (A1) and (A3), (i) follows as in the proof of Theorem 4.1. A straightforward computation gives (8.10).

Theorems 4.2 and and 4.3 now yield the following result. 
Theorem 8.2. Assume (B2)-(B6) hold, and a, $m, \mathscr{R}(\cdot, \mu)$ are defined by $(8.6)-(8.8)$, respectively. Then $\mathscr{R}(\cdot, \mu)$ attains its infimum on $H^{1}(\Omega)$ and,

(i) the value of the problem $\left(\mathcal{R}_{\mu}\right)$ is $\alpha(\mu)=\left\{\begin{array}{ll}0 & \text { if } \mu \leq \lambda_{1} \\ -\frac{1}{2}\left(\mu-\lambda_{1}\right)^{2} & \text { if } \mu>\lambda_{1}\end{array}\right.$.

(ii) When $\mu \leq \lambda_{1}$, the minimizer of $\mathscr{R}(\cdot, \mu)$ is 0 . When $\mu>\lambda_{1}$, the minimizers of $\mathscr{R}(\cdot, \mu)$ are eigenfunctions $\tilde{u}$ of $(a, m)$ corresponding to $\lambda_{1}$ with $\int_{\Omega} m_{0} \tilde{u}^{2} \mathrm{~d} x=\mu-\lambda_{1}$.

As in Section 6, the following unconstrained variational principle can be used to obtain the second eigenvalue and corresponding eigenfunctions of (8.3).

Let $e_{1}$ be an $m$-normalized eigenfunction corresponding to the first eigenvalue $\lambda_{1}$ of (8.3). Consider the problem $\left(\mathcal{R}_{\mu, \tau}\right)$ of minimizing the functional $\mathscr{R}_{\tau}(\cdot, \mu)$ defined on $H^{1}(\Omega)$ by

$$
\mathscr{R}_{\tau}(u, \mu):=\mathscr{R}(u, \mu)+\tau m\left(e_{1}, u\right)^{2}
$$

with $\tau \in(0, \infty)$, and finding

$$
\alpha(\mu, \tau)=\inf _{u \in H^{1}(\Omega)} \mathscr{R}_{\tau}(u, \mu) .
$$

Let $\mu_{c}:=\min \left\{\lambda_{1}+2 \tau, \lambda_{2}\right\}$, and denote by $\tilde{\lambda}_{j}$ the distinct eigenvalues of (8.3) and by $m_{j}$ the multiplicity of $\tilde{\lambda}_{j}$, as done similarly in previous sections. The results for this unconstrained variational principle for finding the second Robin eigenvalue and associated eigenfunctions may be summarized as follows.

Theorem 8.3. Assume (B2)-(B6) hold, the sets $C\left(\tilde{\lambda}_{j} ; \mu\right)$ are given by $(4.7)$, and a, $m, \mathscr{R}_{\tau}(\cdot, \mu)$ are defined by (8.6), (8.7), and (8.11), respectively.

(i) If $0<\mu<\mu_{c}$ and $\lambda_{1}$ is a simple eigenvalue of $(a, m)$, then 0 minimizes $\mathscr{R}_{\tau}(\cdot, \mu)$ on $H^{1}(\Omega)$ and $\alpha(\mu, \tau)=0$.

(ii) If $\mu>\mu_{c}, \tau>\lambda_{2}-\lambda_{1}$ and $\lambda_{1}$ is a simple eigenvalue of $(a, m)$, then the minimizers of $\mathscr{R}_{\tau}(\cdot, \mu)$ on $H^{1}(\Omega)$ are the functions in $C\left(\tilde{\lambda}_{2} ; \mu\right)$ and

$$
\alpha(\mu, \tau)=-\frac{1}{2}\left(\mu-\tilde{\lambda}_{2}\right)^{2}>\alpha(\mu) .
$$

(iii) If $\mu>\mu_{c}$ and $\lambda_{1}$ is an eigenvalue of multiplicity $m_{1} \geq 2$, then the minimizers of $\mathscr{R}_{\tau}(\cdot, \mu)$ on $H^{1}(\Omega)$ are the functions $u$ in $C\left(\tilde{\lambda}_{1} ; \mu\right)$ that also satisfy $m\left(u, e_{1}\right)=0$. In this case, $\alpha(\mu, \tau)=\alpha(\mu)$.

Proof. Lemma 7.1 in [6] shows $m$ is strictly positive on an infinite dimensional subspace of $H^{1}(\Omega)$, so that there is a second distinct eigenvalue for (8.3). The proof is then similar to that of Theorem 6.1 as the pair of bilinear forms $(a, m)$ satisfy $(\mathrm{A} 1)-(\mathrm{A} 3)$.

To enable a Morse index theory for the functional $\mathscr{R}(\cdot, \mu)$ in $(8.8)$, the next lemma shows that the functional has a well-defined second derivative which may be computed just as in Theorem 4.1.

Lemma 8.4. Assume (B2)-(B6) hold and a, $m, \mathscr{R}(\cdot, \mu)$ are defined as above. Then $\mathscr{R}(\cdot, \mu)$ is twice Gâteaux differentiable on $H^{1}(\Omega)$ with second variation at $u$ given by

$$
\delta^{2} \mathscr{R}(u ; v, w ; \mu)=2 a(v, w)+2[m(u, u)-\mu] m(v, w)+4 m(u, v) m(u, w) .
$$

To describe the degeneracy of critical points of $\mathscr{R}(\cdot, \mu)$ and to compute their Morse index, we shall require the weight function $m_{0}$ in (B6) to also obey

$$
\int_{\Omega} m_{0} u^{2} \mathrm{~d} x>0 \quad \text { for all non-zero } u \in H^{1}(\Omega) .
$$

That is, we shall require the bilinear form $m$ in (8.7) to also satisfy (A4). When this holds, taking $b$ in (M1) and (M2) equal to the Hessian form $(v, w) \mapsto \delta^{2} \mathscr{R}(u ; v, w ; \mu)$ defined by (8.13) allows the use of Theorem 3.1 to obtain the following results. As in Section 5 let $\sigma(a, m)$ be the spectrum of $(a, m)$. 
Theorem 8.5. Assume (B2)-(B6), (8.14) hold, and $\mathscr{R}(\cdot, \mu)$ is defined by (8.8). Then

(i) 0 is a non-degenerate critical point of $\mathscr{R}(\cdot, \mu)$ if and only if $\mu$ is not an eigenvalue of (8.3). When $\mu<\tilde{\lambda}_{1}$, the Morse index of 0 is $i(0 ; \mu)=0$, and when $\mu>\tilde{\lambda}_{1}$ then $i(0 ; \mu)=\sum_{\tilde{\lambda}_{k}<\mu} m_{k}$ if $\mu \notin \sigma(a, m)$. When 0 is a degenerate critical point of $\mathscr{R}\left(\cdot, \tilde{\lambda}_{j}\right)$, then its null index is $i_{0}\left(0 ; \tilde{\lambda}_{j}\right)=m_{j}$.

(ii) When $\mu>\tilde{\lambda}_{j}$, then $\tilde{u}_{j}=\left(\mu-\tilde{\lambda}_{j}\right)^{1 / 2} e$, with e an m-normalized eigenfunction corresponding to $\tilde{\lambda}_{j}$, is a non-degenerate critical point of $\mathscr{R}(\cdot, \mu)$ if and only if $\tilde{\lambda}_{j}$ is a simple eigenvalue of $(a, m)$. In this case, the Morse index of $\tilde{u}_{j}$ is $i\left(\tilde{u}_{j} ; \mu\right)=\sum_{k=1}^{j-1} m_{k}$. If $\tilde{\lambda}_{j}$ has multiplicity $m_{j} \geq 2$, then $\tilde{u}_{j}$ is a degenerate critical point of $\mathscr{R}(\cdot, \mu)$ that has null index $i_{0}\left(\tilde{u}_{j} ; \mu\right)=m_{j}-1$.

Proof. As mentioned above, when the function $m_{0}$ also satisfies $(8.14)$ the Hessian form $\delta^{2} \mathscr{R}(u ; \cdot, \cdot, \mu)$ in Lemma 8.4 satisfies (M1) and (M2), so the proofs of Theorems 5.1 and 5.2 yield the desired results as Theorem 3.1 holds for $\delta^{2} \mathscr{R}(u ; \cdot, \cdot, \mu)$.

From the above results, the bifurcation diagram in Figure 1 can be used for the Robin eigenproblem to obtain a bifurcation description for the functional $\mathscr{R}(\cdot, \mu)$ in (8.8) as discussed at the end of Section 4 and also at the end of Section 5.

If we assume that the coefficient function $m_{0}$ satisfies, (B7) $m_{0} \in L^{\infty}(\Omega)$ and there is a constant $k_{4}$ such that $m_{0}(x) \geq k_{4}>0$ for all $x \in \Omega$.

then the results in Theorem 8.5 also follow as the bilinear form $m$ satisfies (A4). Note that Theorem 7.2 in [6] shows that in this case the sequence of eigenfunctions of (8.3) forms also a basis of $L^{2}(\Omega)$.

The results obtained here for Robin eigenproblems parallel those obtained in ([5], Sect. 8) for linear, secondorder, elliptic, boundary value eigenproblems with homogeneous Dirichlet condition.

\section{UNCONSTRAINED VARIATIONAL PRINCIPLES FOR STEKLOV EIGENVALUE PROBLEMS}

A Steklov eigenproblem for a homogeneous linear elliptic partial differential equation is one where the eigenparameter appears only in the boundary condition. Let $\Omega$ be a region in $\mathbb{R}^{N}$ with boundary $\partial \Omega$ that satisfies (B1) and (B2).

This section will analyze an unconstrained variational principle for uniformly elliptic second order equations of the form

$$
L u(x):=-\operatorname{div}(A(x) \nabla u(x))+c(x) u(x)=0 \quad \text { on } \Omega
$$

subject to

$$
B u(x):=(A(x) \nabla u(x)) \cdot \nu(x)+b(x) u(x)=\lambda \rho(x) u(x) \quad \text { on } \partial \Omega .
$$

The boundary weight function $\rho: \partial \Omega \rightarrow[0, \infty]$ is assumed to be Borel-measurable on $\partial \Omega$ and is said to be normalized provided

$$
\int_{\partial \Omega} \rho \mathrm{d} \sigma=1
$$

Here the interest is in finding non-trivial solutions $(\lambda, u) \in \mathbb{R} \times H^{1}(\Omega)$ satisfying

$$
\int_{\Omega}[(A \nabla u) \cdot \nabla v+c u v] \mathrm{d} x+\int_{\partial \Omega} b u v \mathrm{~d} \sigma=\lambda \int_{\partial \Omega} \rho u v \mathrm{~d} \sigma \text { for all } v \in H^{1}(\Omega) .
$$

This is a weak form of the system (9.1) and (9.2). The requirement that $\rho$ be normalized fixes a scaling of the Steklov eigenvalues. This is an eigenproblem of the type introduced in Section 2 with $V=H^{1}(\Omega), a(\cdot, \cdot)$ defined by $(8.6)$, and $m(\cdot, \cdot)$ defined by

$$
m(u, v):=\int_{\partial \Omega} \rho u v \mathrm{~d} \sigma
$$


The Borel measurable function $\rho$ is required to satisfy,

(B8) $\rho \in L^{\infty}(\partial \Omega, \mathrm{d} \sigma)$ is normalized and $\rho \geq \rho_{0}>0, \sigma$ a.e. on $\partial \Omega$.

When (B2) and (B8) hold, $m$ will satisfy (A2), (A3). Then Lemma 8.1 and Theorem 8.2 of [6] yield the existence of an increasing sequence of strictly positive Steklov eigenvalues $\Lambda:=\left\{\lambda_{j}: j \in \mathbb{N}\right\}$, with $\lim _{j \rightarrow \infty} \lambda_{j}=$ $\infty$, and a corresponding sequence $\mathcal{E}:=\left\{e_{j}: j \in \mathbb{N}\right\}$ of eigenfunctions of (9.4). In particular, there is a least positive eigenvalue $\lambda_{1}$ and a corresponding eigenvector of (9.4).

Consider the parametrized functional $\mathscr{S}: H^{1}(\Omega) \times(0, \infty) \rightarrow \mathbb{R}$ defined by

$$
\mathscr{S}(u, \mu):=\int_{\Omega}\left[(A \nabla u) \cdot \nabla u+c u^{2}\right] \mathrm{d} x+\int_{\partial \Omega}(b-\mu \rho) u^{2} \mathrm{~d} \sigma+\frac{1}{2}\left[\int_{\partial \Omega} \rho u^{2} \mathrm{~d} \sigma\right]^{2}
$$

The variational principle $\left(\mathcal{S}_{\mu}\right)$ is to minimize $\mathscr{S}(\cdot, \mu)$ on $H^{1}(\Omega)$ and find

$$
\alpha(\mu):=\inf _{u \in H^{1}(\Omega)} \mathscr{S}(u, \mu) .
$$

The following lemma describes the essential properties of $\mathscr{S}(\cdot, \mu)$ for our analyses.

Lemma 9.1. Assume (B2)-(B5), (B8) hold, and $a, m, \mathscr{S}$ are defined by (8.6), (9.5), (9.6), respectively. Then

(i) $\mathscr{S}(\cdot, \mu)$ is continuous, coercive and weakly l.s.c. on $H^{1}(\Omega)$,

(ii) $\mathscr{S}(\cdot, \mu)$ is Gâteaux differentiable on $H^{1}(\Omega)$ with first variation at u given by

$$
\delta \mathscr{S}(u ; v ; \mu)=2 a(u, v)+2\left[\int_{\partial \Omega} \rho u^{2} \mathrm{~d} \sigma-\mu\right] m(u, v)
$$

in the direction of the function $v$ in $H^{1}(\Omega)$, and

(iii) $\mathscr{S}(\cdot, \mu)$ is twice Gâteaux differentiable with second variation at $u$ in the directions $v, w$ given by

$$
\delta^{2} \mathscr{S}(u ; v, w ; \mu)=2 a(v, w)+2[m(u, u)-\mu] m(v, w)+4 m(u, v) m(u, w) .
$$

Proof. As the pair of bilinear forms $(a, m)$ satisfy (A1)-(A3), (i) follows as in the proof of Theorem 4.1. Straightforward computations yield (9.8) and (9.9).

Theorems 4.2 and 4.3 now yield the following result.

Theorem 9.2. Assume (B2)-(B5), (B8) hold, and a, m, S are defined by (8.6), (9.5), (9.6), respectively. Then $\mathscr{S}(\cdot, \mu)$ attains its infimum on $H^{1}(\Omega)$ and,

(i) the value of the problem $\left(\mathcal{S}_{\mu}\right)$ is $\alpha(\mu)=\left\{\begin{array}{ll}0 & \text { if } \mu \leq \lambda_{1} \\ -\frac{1}{2}\left(\mu-\lambda_{1}\right)^{2} & \text { if } \mu>\lambda_{1}\end{array}\right.$.

(ii) When $\mu \leq \lambda_{1}$, the minimizer of $\mathscr{S}(\cdot, \mu)$ is 0 . When $\mu>\lambda_{1}$, the minimizers of $\mathscr{S}(\cdot, \mu)$ are eigenfunctions $\tilde{u}$ of $(a, m)$ corresponding to $\lambda_{1}$ with $\int_{\Omega} \rho \tilde{u}^{2} \mathrm{~d} x=\mu-\lambda_{1}$.

As stated in Lemma 8.1, when (B2)-(B5) hold, $a(\cdot, \cdot)$ defines an equivalent inner product on $H^{1}(\Omega)$. A function $u \in H^{1}(\Omega)$ is a weak solution of the equation $L u=0$ on $\Omega$ provided

$$
a(u, v)=0 \text { for all } v \in H_{0}^{1}(\Omega) .
$$

The subspace of all such functions will be denoted $N(L)$ and this definition yields the $a$-orthogonal decomposition

$$
H^{1}(\Omega)=H_{0}^{1}(\Omega) \oplus_{a} N(L),
$$


with $N(L)$ being a closed subspace of $H^{1}(\Omega)$. Moreover when (B8) holds then $m(\cdot, \cdot)$ defines an inner product on $N(L)$; this is called the $\rho$-inner product on $N(L)$. The Steklov eigenfunctions of (9.4) may be chosen to be a $\rho$-orthogonal basis of $N(L)$ from Theorem 8.2 in [6].

To provide a Morse index theory for the Steklov eigenproblem, we therefore restrict the domain of the pair of bilinear forms $(a, m)$ to the closed subspace $N(L)$ of $H^{1}(\Omega)$.

As in the last section, the $\sigma(a, m)$ denotes the collection of distinct (strictly positive) eigenvalues $\tilde{\lambda}_{j}$ of $(9.4)$, and the multiplicity of the $j$ th distinct eigenvalue $\tilde{\lambda}_{j}$ is $m_{j}$.

Theorem 9.3. Assume (B2)-(B5), (B8) hold, and a, $m, \mathscr{S}(\cdot, \mu)$ are defined by (8.6), (9.5), (9.6), respectively, on the subspace $N(L)$ of $H^{1}(\Omega)$. Then,

(i) 0 is a non-degenerate critical point of $\mathscr{S}(\cdot, \mu)$ if and only if $\mu$ is not an eigenvalue of (9.4). When $\mu<\tilde{\lambda}_{1}$, the Morse index of 0 is $i(0 ; \mu)=0$, and when $\mu>\lambda_{1}$ then $i(0 ; \mu)=\sum_{\tilde{\lambda}_{k}<\mu} m_{k}$ if $\mu \notin \sigma(a, m)$. When 0 is a degenerate critical point of $\mathscr{S}\left(\cdot, \tilde{\lambda}_{j}\right)$, then its null index is $i_{0}\left(0 ; \tilde{\lambda}_{j}\right)=m_{j}$.

(ii) When $\mu>\tilde{\lambda}_{j}$, then $\tilde{u}_{j}=\left(\mu-\tilde{\lambda}_{j}\right)^{1 / 2} e$, with e an m-normalized eigenfunction corresponding to $\tilde{\lambda}_{j}$, is a non-degenerate critical point of $\mathscr{S}(\cdot, \mu)$ if and only if $\tilde{\lambda}_{j}$ is a simple eigenvalue of (9.4). In this case, the Morse index of $\tilde{u}_{j}$ is $i\left(\tilde{u}_{j} ; \mu\right)=\sum_{k=1}^{j-1} m_{k}$. If $\tilde{\lambda}_{j}$ has multiplicity $m_{j} \geq 2$, then $\tilde{u}_{j}$ is a degenerate critical point of $\mathscr{S}(\cdot, \mu)$ that has null index $i_{0}\left(\tilde{u}_{j} ; \mu\right)=m_{j}-1$.

Proof. When $H^{1}(\Omega)$ is replaced by $N(L)$, the results of Lemma 9.1 and Theorem 9.2 hold for the functional $\mathscr{S}(\cdot, \mu): N(L) \rightarrow \mathbb{R}$ defined by (9.6) as $N(L)$ is a closed subspace of $H^{1}(\Omega)$ and the Steklov eigenfunctions of (9.4) are in $N(L)$. Moreover, the bilinear form $m$ in (9.5) is strictly positive on $N(L)$, so that Theorem 3.1 gives the desired results as in the proof of Theorems 5.1 and 5.2 .

As in the Robin eigenproblem, Figure 1 and the bifurcation results at the end of Section 4 and Section 5 apply to the Steklov eigenproblem, where the schematic diagram is, in this case, on the space $(0, \infty) \times N(L)$ instead of $(0, \infty) \times H^{1}(\Omega)$.

\section{VARIATIONAL PRINCIPLES FOR INDEFINITE STEKLOV EIGENPROBLEMS}

Suppose now that one wants to investigate the eigenvalues of (9.4) with the boundary weight function $\rho$ allowed to change sign on $\partial \Omega$. We shall require

$$
\rho:=\rho_{+}-\rho_{-} \text {is a Borel function on } \partial \Omega \text { that is } \sigma \text {-essentially bounded on } \partial \Omega \text { with }
$$

$$
0<\int_{\partial \Omega} \rho_{+} \mathrm{d} \sigma<\int_{\partial \Omega}|\rho| \mathrm{d} \sigma=1
$$

Let $a, m, \mathscr{S}$ be the forms and functionals defined in the preceding section and define $\mathscr{S}_{-}: H^{1}(\Omega) \times(0, \mu) \rightarrow \mathbb{R}$ by

$$
\mathscr{S}_{-}(u, \mu):=a(u, u)+\mu m(u, u)+\frac{1}{2} m(u, u)^{2}
$$

Consider the problems $\left(\mathcal{S}_{\mu+}\right)$ and $\left(\mathcal{S}_{\mu-}\right)$ of minimizing $\mathscr{S}(\cdot, \mu)$ and $\mathscr{S}_{-}(\cdot, \mu)$ on $H^{1}(\Omega)$. Let

$$
\alpha_{+}(\mu):=\inf _{u \in H^{1}(\Omega)} \mathscr{S}(u, \mu) \quad \text { and } \quad \alpha_{-}(\mu):=\inf _{u \in H^{1}(\Omega)} \mathscr{S}_{-}(u, \mu) .
$$

As in Section 7, results in [6] may be used to yield the existence of eigenvalues

$$
\cdots \leq \lambda_{2}^{-} \leq \lambda_{1}^{-}<0<\lambda_{1}^{+} \leq \lambda_{2}^{+} \leq \ldots
$$

in the case (B2)-(B5), (B9) hold. One sees that Lemma 9.1 holds when (B9) replaces (B8), so the following result holds. 
Theorem 10.1. Assume (B2)-(B5), (B9) hold with a, m, $\mathscr{S}, \mathscr{S}_{-}$defined by (8.6), (9.5), (9.6), (10.1) respectively. Then $\mathscr{S}(\cdot, \mu)$ and $\mathscr{S}_{-}(\cdot, \mu)$ attain their infima on $H^{1}(\Omega)$,

(i) the value of the problem $\left(\mathcal{S}_{\mu+}\right)$ is $\alpha_{+}(\mu)=\left\{\begin{array}{ll}0 & \text { if } \mu \leq \lambda_{1}^{+} \\ -\frac{1}{2}\left(\mu-\lambda_{1}^{+}\right)^{2} & \text { if } \mu>\lambda_{1}^{+}\end{array}\right.$, and

(ii) the value of the problem $\left(\mathcal{S}_{\mu-}\right)$ is $\alpha_{-}(\mu)=\left\{\begin{array}{ll}0 & \text { if } \mu \leq-\lambda_{1}^{-} \\ -\frac{1}{2}\left(\mu+\lambda_{1}^{-}\right)^{2} & \text { if } \mu>-\lambda_{1}^{-}\end{array}\right.$.

(iii) When $\mu \leq \lambda_{1}^{+}$(respectively, $\mu \leq-\lambda_{1}^{-}$), the minimizer of $\mathscr{S}(\cdot, \mu)$ (respectively, the minimizer of $\mathscr{S}_{-}(\cdot, \mu)$ ) is 0 .

When $\mu>\lambda_{1}^{+}$(respectively, $\mu>-\lambda_{1}^{-}$), the minimizers of $\mathscr{S}(\cdot, \mu)$ (respectively, $\mathscr{S}_{-}(\cdot, \mu)$ ) are eigenfunctions $\tilde{u}$ of $(a, m)$ corresponding to $\lambda_{1}^{+}$(respetively, $\lambda_{1}^{-}$) satisfying $\int_{\partial \Omega} \rho \tilde{u}^{2} \mathrm{~d} \sigma=\mu-\lambda_{1}^{+}$(respectively, $\int_{\partial \Omega} \rho \tilde{u}^{2} \mathrm{~d} \sigma=$ $\left.-\mu-\lambda_{1}^{-}\right)$.

Proof. The proof of this follows in much the same way as that of Theorem 7.1.

More generally for large $\mu$ there may be multiple critical points of $\mathscr{S}_{-}(\cdot, \mu)$ on $H^{1}(\Omega)$ and these will be (Steklov) eigenfunctions of (9.3) associated with negative eigenvalues $\lambda$.

A computation analogous to Theorem 9.3 of the Morse indices of the non-degenerate critical points of these functionals may be performed.

\section{UNCONSTRAINED VARIATIONAL PRINCIPLES FOR GENERAL EIGENVALUE PROBLEMS}

Recently there has been interest in elliptic eigenproblems where the eigenvalue appears in both the differential equation and in the boundary conditions. See Auchmuty [6], Section 11 and the references cited there and Belinskiy [9].

In [6] various constrained variational problems for these eigenproblems were studied - based on the use of bilinear and quadratic forms on Sobolev spaces rather than linear operators. Here analogous unconstrained variational problems will be described and analyzed

A model problem for this is to find non-trivial solutions $(\lambda, u)$ of

$$
-\operatorname{div}(A(x) \nabla u(x))+c(x) u(x)=\lambda m_{0}(x) u(x) \quad \text { on } \Omega
$$

subject to

$$
(A(x) \nabla u(x)) \cdot \nu(x)+b(x) u(x)=\lambda \rho(x) u(x) \quad \text { on } \partial \Omega .
$$

Here the eigenparameter $\lambda$ appears in both the equation and boundary condition.

The weak form of (11.1), (11.2) is to find non-trivial solutions $(\lambda, u) \in \mathbb{R} \times H^{1}(\Omega)$ that satisfy, for all $v \in H^{1}(\Omega)$,

$$
\int_{\Omega}[(A \nabla u) \cdot \nabla v+c u v] \mathrm{d} x+\int_{\partial \Omega} b u v \mathrm{~d} \sigma=\lambda\left[\int_{\Omega} m_{0} u v \mathrm{~d} x+\int_{\partial \Omega} \rho u v \mathrm{~d} \sigma\right] .
$$

This is an $(a, m)$ eigenproblem with $V=H^{1}(\Omega), a$ as in (8.6) and $m$ defined by

$$
m(u, v):=\int_{\Omega} m_{0} u v \mathrm{~d} x+\int_{\partial \Omega} \rho u v \mathrm{~d} \sigma .
$$

When (B7) and (B8) hold, the bilinear form $m$ satisfies (A2), (A4); this follows from Lemma 9.1 of [6]. In particular, there is a least positive eigenvalue $\lambda_{1}$ of (11.3). Consider the functional $\mathscr{L}: H^{1}(\Omega) \times(0, \infty) \rightarrow \mathbb{R}$ defined by

$$
\mathscr{L}(u, \mu):=\int_{\Omega}\left[(A \nabla u) \cdot \nabla u+\left(c-\mu m_{0}\right) u^{2}\right] \mathrm{d} x+\int_{\partial \Omega}(b-\mu \rho) u^{2} \mathrm{~d} \sigma+\frac{1}{2} m(u, v)^{2} .
$$


The unconstrained variational principle $\left(\mathcal{L}_{\mu}\right)$ is to minimize $\mathscr{L}(\cdot, \mu)$ on $H^{1}(\Omega)$ and find

$$
\alpha(\mu)=\inf _{u \in H^{1}(\Omega)} \mathscr{L}(u, \mu) .
$$

The essential properties of this functional $\mathscr{L}(\cdot, \mu)$ include the following which is proved in the same way as Lemma 9.1.

Lemma 11.1. Assume (B2)-(B6), (B8) hold, and a,m,L्L are defined by (8.6), (11.4), (11.5), respectively. Then:

(i) $\mathscr{L}(\cdot, \mu)$ is continuous, coercive and weakly l.s.c. on $H^{1}(\Omega)$,

(ii) $\mathscr{L}(\cdot, \mu)$ is Gâteaux differentiable on $H^{1}(\Omega)$ with first variation at u given by

$$
\delta \mathscr{S}(u ; v ; \mu)=2 a(u, v)+2[m(u, u)-\mu] m(u, v)
$$

in the direction of the function $v$ in $H^{1}(\Omega)$, and

(iii) $\mathscr{L}(\cdot, \mu)$ is twice Gâteaux differentiable with second variation at $u$ in the directions $v, w$ given by

$$
\delta^{2} \mathscr{L}(u ; v, w ; \mu)=2 a(v, w)+2[m(u, u)-\mu] m(v, w)+4 m(u, v) m(u, w) .
$$

Theorems 4.2 and and 4.3 now yield the following result.

Theorem 11.2. Assume (B2)-(B5), (B8) hold, and a, $m, \mathscr{L}$ are defined by (8.6), (11.4), (11.5), respectively. Then $\mathscr{L}(\cdot, \mu)$ attains its infimum on $H^{1}(\Omega)$ and,

(i) the value of the problem $\left(\mathcal{L}_{\mu}\right)$ is $\alpha(\mu)=\left\{\begin{array}{ll}0 & \text { if } \mu \leq \lambda_{1} \\ -\frac{1}{2}\left(\mu-\lambda_{1}\right)^{2} & \text { if } \mu>\lambda_{1}\end{array}\right.$.

(ii) When $\mu \leq \lambda_{1}$, the minimizer of $\mathscr{L}(\cdot, \mu)$ is 0 . When $\mu>\lambda_{1}$, the minimizers of $\mathscr{L}(\cdot, \mu)$ are eigenfunctions $\tilde{u}$ of $(a, m)$ corresponding to $\lambda_{1}$ with $\int_{\Omega} m_{0} \tilde{u}^{2} \mathrm{~d} x+\int_{\partial \Omega} \rho \tilde{u}^{2} \mathrm{~d} \sigma=\mu-\lambda_{1}$.

Proof. As the bilinear forms $a$ and $m$ satisfy (A1) - (A4), the proof is similar to that of Theorems 4.2 and 4.3.

When $\mu>\lambda_{1}$, the functional $\mathscr{L}(\cdot, \mu)$ has multiple critical points. The following result describes the Morse indices of the critical points of this functional. As before, let $\sigma(a, m)$ denote the collection of distinct strictly positive eigenvalues of (11.3) and let the multiplicity of the $j$ th distinct eigenvalue $\tilde{\lambda}_{j}$ be denoted $m_{j}$.

Theorem 11.3. Assume (B2)-(B6), (B8), (8.14) hold, and a,m,L्L are defined by (8.6), (11.4), (11.5), respectively. Then,

(i) 0 is a nondegenerate critical point of $\mathscr{L}(\cdot, \mu)$ if and only if $\mu$ is not an eigenvalue of (11.3). When $\mu<\tilde{\lambda}_{1}$, the Morse index of 0 is $i(0 ; \mu)=0$, and when $\mu>\tilde{\lambda}_{1}$ then $i(0 ; \mu)=\sum_{\tilde{\lambda}_{k}<\mu} m_{k}$ if $\mu \notin \sigma(a, m)$. When 0 is a degenerate critical point of $\mathscr{L}\left(\cdot, \tilde{\lambda}_{j}\right)$, then its null index is $i_{0}\left(0 ; \tilde{\lambda}_{j}\right)=m_{j}$.

(ii) When $\mu>\tilde{\lambda}_{j}$, then $\tilde{u}_{j}=\left(\mu-\tilde{\lambda}_{j}\right)^{1 / 2}$ e, with e an m-normalized eigenfunction corresponding to $\tilde{\lambda}_{j}$, is a non-degenerate critical point of $\mathscr{L}(\cdot, \mu)$ if and only if $\tilde{\lambda}_{j}$ is a simple eigenvalue of (11.3). In this case, the Morse index of $\tilde{u}_{j}$ is $i\left(\tilde{u}_{j} ; \mu\right)=\sum_{k=1}^{j-1} m_{k}$. If $\tilde{\lambda}_{j}$ has multiplicity $m_{j} \geq 2$, then $\tilde{u}_{j}$ is a degenerate critical point of $\mathscr{L}(\cdot, \mu)$ that has null index $i_{0}\left(\tilde{u}_{j} ; \mu\right)=m_{j}-1$.

Proof. Since the bilinear form $m$ in (11.4) satisfies (A2) and (A4), and since the Hessian form $(v, w) \mapsto$ $\delta^{2} \mathscr{L}(u ; v, w ; \mu)$ given by 11.8 satisfies $(\mathrm{M} 1)$ at critical points of $\mathscr{L}(\cdot, \mu)$, the results follow as in the proof of Theorems 5.1 and 5.2 . 


\section{REFERENCES}

[1] H. Attouch, G. Buttazzo and G. Michaille, Variational Analysis in Sobolev and BV Spaces: Applications to PDEs and Optimization. MPS-SIAM, Philadelphia (2005).

[2] G. Auchmuty, Dual Variational Principles for Eigenvalue Problems, in Nonlinear Functional Analysis and its Applications Part 1, edited by F.E. Browder. Vol. 45 of Proc. Symp. Pure Math. AMS (1986) 55-72.

[3] G. Auchmuty, Unconstrained Variational Principles for Eigenvalues of Real Symmetric Matrices. SIAM J. Appl. Math 20 (1989) 1186-1207.

[4] G. Auchmuty, Variational Principles for Eigenvalues of Compact Operators, SIAM J. Appl. Math. 20 (1989) $1321-1335$.

[5] G. Auchmuty, Variational Principles for Self-Adjoint Elliptic Eigenproblems, in Nonsmooth/Nonconvex Mechanics. Edited by Gao, Ogden and Stavroulakis. Kluwer, Dordrecht (2001) 15-42.

[6] G. Auchmuty, Bases and Comparison Results for Linear Elliptic Eigenproblems. J. Math. Anal. Appl. 390 (2012) $394-406$.

[7] F. Belgacem, Elliptic Boundary Value Problems with indefinite weights. Vol. 368 of Research Notes Math. Pitman (1997).

[8] P. Blanchard and E. Brüning, Variational Methods in Mathematical Physics. Springer-Verlag, Berlin (1992).

[9] B. Belinskiy, Eigenvalue problems for Elliptic type partial differential operators with spectral parameter contained linearly in boundary conditions, in Proc. of Partial Differential and Integral Equations. Edited by Begehr. Kluwer (1999).

[10] D.E. Edmunds and W.D. Evans, Spectral Theory and Differential Operators. Oxford University Press, Oxford (1987).

[11] E. Zeidler, Nonlinear Functional Analysis and its Applications, II/A, Linear Monotone Operators. Springer Verlag, New York (1990).

[12] E. Zeidler, Nonlinear Functional Analysis and its Applications, III, Variational Methods Operators. Springer Verlag, New York (1985). 